OPEN ACCESS

Edited by:

Scott D. Moore,

Duke University, United States

Reviewed by:

Simona Lattanzi,

Marche Polytechnic University, Italy

Jiguang Wang,

Shanghai Jiao Tong University, China

*Correspondence:

Yunling Zhang

yunlingzhang2004@163.com

${ }^{\dagger}$ These authors have contributed equally to this work

Specialty section: This article was submitted to Neuropharmacology,

a section of the journal

Frontiers in Pharmacology

Received: 16 November 2021 Accepted: 18 January 2022

Published: 14 February 2022

Citation:

Shen W, Fan $X$, Wang $L$ and Zhang $Y$ (2022) Traditional Chinese Medicine for Post-Stroke Cognitive Impairment: A Systematic Review and Meta-Analysis. Front. Pharmacol. 13:816333. doi: 10.3389/fphar.2022.816333

\section{Traditional Chinese Medicine for Post-Stroke Cognitive Impairment: A Systematic Review and Meta-Analysis}

\author{
Wei Shen ${ }^{\dagger}$, Xueming Fan ${ }^{\dagger}$, Liuding Wang and Yunling Zhang* \\ Xiyuan Hospital, China Academy of Chinese Medical Sciences, Beijing, China
}

Background: Post-stroke cognitive impairment (PSCl) affects more than one-third of stroke patients, and causes much greater harm to long-term function than the initial brain damage. No conventional Western medications have shown convincing clinical effectiveness for treating PSCI. Research shows that Traditional Chinese medicine (TCM) can improve cognitive function in patients. However, the clinical efficacy and safety remain controversial. The aim of this study was to examine the effectiveness and harmful effects of TCMs in the treatment of PSCl.

Method: We searched seven databases and two clinical registration websites for randomized controlled trials (RCTs). The revised Cochrane risk of bias tool (RoB 2.0) was used to evaluate the methodological quality and RevMan 5.4 was used for data analysis. This study has been submitted to PROSPERO with registration number is CRD42020149299.

Results: We included 34 studies in this review. The results of this study showed that TCM adjuvant therapy improved scores on the $\mathrm{MoCA}[\mathrm{MD}=2.55,95 \% \mathrm{Cl}(1.56,3.53), p<0.00001$; $\mathrm{MD}=3.07,95 \% \mathrm{Cl}(1.98,4.17), p<0.00001$ at treatment duration of $<3$ and 3 months, respectively], MMSE [MD $=2.55,95 \% \mathrm{Cl}(1.99,3.10), p<0.00001 ; \mathrm{MD}=2.53,95 \% \mathrm{Cl}(1.59$, 3.47), $p<0.00001 ; \mathrm{MD}=2.91,95 \% \mathrm{Cl}(1.26,4.56), p=0.0006 ; \mathrm{MD}=3.11,95 \% \mathrm{Cl}(-0.04$, 6.27), $p=0.05$ at treatment duration of $<3,3,4$, and 6 months, respectively], and $\mathrm{BI}[\mathrm{MD}=7.34$, $95 \% \mathrm{Cl}(3.83,10.85), p<0.0001 ; \mathrm{MD}=8.98,95 \% \mathrm{Cl}(4.76,13.21), p<0.0001$ at treatment duration of $\angle 3$ and 3 months, respectively] and reduced scores on the ADL (MD $=-8.64,95 \%$ $\mathrm{Cl}(-9.83,-7.45), p<0.00001 ; \mathrm{MD}=-2.00,95 \% \mathrm{Cl}(-2.94,-1.06), p<0.0001$ at treatment duration of 3 and 4 months, respectively], NIHSS [MD $=-2.48,95 \% \mathrm{Cl}(-4.97,0.00), p=0.05$; $\mathrm{MD}=-3.81,95 \% \mathrm{Cl}(-6.21,-1.40), p=0.002$ at treatment duration of $<3$ and 3 months, respectively], and CSS [MD $=-2.47,95 \% \mathrm{Cl}(-3.49,-1.45), p<0.00001$ at a treatment duration of 3 months]. No serious adverse reactions were observed.

Conclusion: Despite the significant positive results, the present evidence supports, to a limited extent because of the methodological flaws and herbal heterogeneity, that TCM adjuvant therapy can be used for patients with PSCl. While, further rigorous RCTs are warranted to confirm the efficacy and safety of TCM.

Systematic Review Registration: https://www.crd.york.ac.uk/prospero, identifier CRD42020149299.

Keywords: traditional Chinese medicine, post-stroke cognitive impairment, systematic review, cognitive function, potential mechanism 


\section{INTRODUCTION}

Post-stroke cognitive impairment (PSCI) is one of the main complications of stroke (Mijajlović et al., 2017), and causes more harm to long-term function than the initial brain injury (Swartz et al., 2016). PSCI can be categorized as post-stroke cognitive impairment no dementia (PSCIND) and post-stroke dementia (PSD), according to the severity of cognitive decline. Previous research indicates that the overall prevalence of PSCI varies from 30\% to 50\% (Mellon et al., 2015), and the incidence of dementia in patients with severe and minor stroke is $34.4 \%$ and $8.2 \%$, respectively (Pendlebury and Rothwell, 2019). PSCI frequently causes severe damage to attention and executive ability (Aam et al., 2020; Zhang and Bi, 2020), affects activities of daily living (Blomgren et al., 2019), and places heavy financial burdens on paramedics and medical institutions (Black et al., 2018). On 4 May 2016, the American Heart Association and the American Stroke Association jointly issued the Guidelines for Adult Stroke Rehabilitation and Recovery, which emphasize the importance of memory and cognitive assessment in post-stroke recuperation. For example, it is recommended that stroke patients receive cognitive function training for post-acute recovery (Class I, Level A evidence), and that all stroke patients should be screened for cognitive deficits before discharge (Class I, Level B evidence) (Gittler and Davis, 2018). Although over one-third of stroke patients may develop PSCI, its underlying pathogenesis remains unclear (Kim et al., 2020a). Various mechanisms may play an important role in PSCI, such as cerebral small vessel disease (Teng et al., 2017), lesions in neuroanatomical structures (Sun et al., 2014), neuroinflammation and oxidative stress (Zhang et al., 2021). Recent study indicated that endovascular treatment can result in better cognitive performance of stroke patients (Lattanzi et al., 2020), while there is still a lack of sufficient evidence to support oral drug therapy in this process (Quinn et al., 2021). Expert consensus recommends the use of drugs for the treatment of vascular cognitive impairment and Alzheimer's disease (Dong et al., 2017; Wang et al., 2021a); however, the restoration of cognitive function or prevention of further decline after stroke are still uncertain (Brainin et al., 2015). Therefore, identification of possible specific treatments plays an important role in the prognosis of PSCI (Wang, 2020a).

In Asia, Traditional Chinese medicine (TCM) is widely used to improve the quality of life of patients with neurodegenerative disease and neuropsychiatric disorders (Feng et al., 2021). Previous research has shown that TCM plays an important role in improving cognitive function in patients. For instance, Danshen extract reduces the contents of tumor necrosis factor- $\alpha$, interleukin- $1 \beta$, and interleukin- 6 in the hippocampus and facilitates learning and memory in rats (Kim et al., 2015); huperzine A, an extract from Huperzia serrata, selectively inhibits acetylcholinesterase activity, increases acetylcholine levels in the brain, and improves cognitive function in patients with dementia ( $\mathrm{Xu}$ et al., 2012; Xing et al., 2014); berberine effectively alleviates amyloid- $\beta$-induced neuroinflammation and regulates amyloid precursor protein metabolism (Jia et al., 2012); and tetramethylpyrazine inhibits angiogenesis and platelet aggregation and improves cerebral microcirculation (Cai et al., 2014; Cui et al., 2015). TCM may be a useful drug therapy to improve cognitive function, but small sample sizes, lack of long-term follow-up, and heterogeneity between studies (Dong et al., 2017) mean that the clinical efficacy and safety of TCM remain controversial. A previous systematic review demonstrated that TCM has a positive effect on cognitive function in PSCI with no serious adverse events (Shen et al., 2020). However, the search strategy of this study was incomplete and several new controlled trials have been conducted since its publication. Therefore, an updated systematic review of the use of TCM for PSCI is needed to provide a firm basis for the treatment of this disease.

\section{METHODS}

\section{Protocol Registration}

This systematic review was registered with the International Prospective Register of Systematic Reviews (PROSPERO) (ID $=$ CRD42020149299).

\section{Search Methods}

A comprehensive retrieval was conducted using the following medical databases: China National Knowledge Infrastructure (CNKI), Wanfang Database, China Science and Technology Journal Database (VIP), and Chinese Biomedical literature Service System (SinoMed) in Chinese, and PubMed, EMBASE, and Cochrane Library in English. The retrieval time was from database inception to February 2021. The search strategy included a combination of medical subject headings and freetext terms. Ongoing or unpublished studies registered on the clinical registration websites (e.g., Chinese Clinical Trial Registry, Clinical Trials.gov) were also searched to obtain more general empirical data. In October 2021, we updated the databases search using the same search method.

\section{Inclusion Criteria Type of Study}

Randomized controlled trials (RCTs) that evaluated the clinical effectiveness and safety of TCM for PSCI were eligible. Studies had to be full-text articles in English or Chinese, regardless of study site, publication date, or study status.

\section{Type of Participant}

We included studies in which patients had a firm diagnosis of PSCI (Wang, 2020a). In addition, because PSCI suggests a causal relationship between stroke and cognitive decline, studies that included a first diagnosis of stroke and a second diagnosis of cognitive impairment were also included.

\section{Type of Intervention}

Included studies featured an intervention group treated with TCM combined with conventional Western medication and a control group that received the same conventional Western 
medication. TCM interventions were defined as prescriptions containing multiple herbs, a single herb, or Chinese patent medicines, with no restrictions on dosage, dosage form, and mode of administration. Conventional Western medication had to feature expert consensus recommended drugs (Dong et al., 2017; Wang, 2020a; Wang et al., 2021a), such as Memantine, Nimodipine, and Oxiracetam. We placed no restriction on the treatment course, but the intervention and control groups had to be the same across studies.

\section{Type of Outcome}

The primary outcome was cognitive assessment using at least one of the internationally recognized assessment scales, such as the Montreal Cognitive Assessment (MoCA), Mini-Mental State Examination (MMSE), Alzheimer's Disease Assessment ScaleCognitive Subscale (ADAS-cog), Vascular Dementia Assessment Scale-Cognitive Subscale (VaDAS-cog), Wechsler Memory Scale (WMS), Hasegawa Dementia Scale (HDS), or Clinical Dementia Rating (CDR).

The secondary outcomes comprised assessment of activities of daily living [using for example the Barthel Index (BI) or the Activities of Daily Living Scale (ADL)] and assessment of neurological deficits [using for example the National Institutes of Health Stroke Scale (NIHSS) or the China Stroke Scale (CSS)].

Safety indicators, such as the incidence of adverse events or adverse reactions, were used to analyze the clinical safety.

\section{Exclusion Criteria}

Studies that used folk medicine and Chinese medicine extracts were excluded, because they were not considered as TCM. Studies that did not clearly identify the drug use or that had incomplete or duplicated data were excluded as well.

\section{Study Selection and Data Extraction}

Endnote X9 (Clarivate Analytics, https://www.endnote.com) was used to manage the literatures. The titles, abstracts, and full text of potentially relevant studies were read and eligible studies were identified. The reasons for exclusion were recorded. Two authors independently conducted the literature screening and information extraction according to a predetermined standardized information extraction table. The data items comprised 1) General information (title, first author, publication date); 2) Study characteristics (sample size, method of randomization, allocation); 3) Participant characteristics (age, gender, course of disease); 4) Intervention characteristics (intervention measures, course of treatment, composition of TCM, follow-ups); 5) Outcomes (outcome measures, adverse events). A third author resolved any disagreements.

\section{Risk of Bias Assessment of Included Studies}

We assessed the methodological quality of the included studies using the revised Cochrane risk of bias tool (RoB 2.0) (Sterne et al., 2019). The evaluation domains comprised randomization process, deviations from intended interventions, missing outcome data, measurement of the outcome, and selection of the reported result. The risk of bias for each domain was evaluated as high risk, low risk and some concerns. Results of the risk of bias assessment were summarized using a risk of bias graph and a risk of bias summary figure. Two authors independently extracted the information. A third author resolved any disagreements.

\section{Data Synthesis and Statistical Analysis}

RevMan 5.4 from the Cochrane Collaboration was used for data analysis. We used the pooled relative risk [and confidence intervals $(\mathrm{CI})$ ] to analyze dichotomous outcomes and the mean difference (MD) or standard mean difference (SMD) (and CI) to analyze continuous outcomes. For some studies that reported only the median, minimum and maximum values, and/or the first and third quartiles, we used a unified approach to estimate the sample mean and standard deviation from these values [Method 1 (Wan et al., 2014; Luo et al., 2018) for normally distributed data, Method 2 (Mcgrath et al., 2020) for non-normally distributed data]. Cochrane's $X^{2}$ test and $I^{2}$ were used to assess heterogeneity. A fixed-effect model was used for meta-analysis if the statistical heterogeneity among the results was not obvious $\left(p>0.1, I^{2}<50 \%\right)$, and a random-effect model was used for meta-analysis if the statistical heterogeneity among the results was obvious ( $\left.p \leq 0.1,50 \% \leq I^{2}<90 \%\right)$. Descriptive analysis was used to summarize the outcomes if the statistical heterogeneity between the results was significant $\left(p \leq 0.1, I^{2} \geq\right.$ $90 \%)$. If the treatment course had a substantial clinical effect, we carried out subgroup analysis to examine differences in the length of therapy. Publication bias was examined using funnel plot analyses if the number of studies was $\geq 10$.

\section{Certainty Assessment of Evidence}

The certainty of evidence for each specific outcome was evaluated by using the Grading of Recommendations, Assessment, Development, and Evaluations (GRADE) system (Guyatt et al., 2008). Two authors separately assessed the quality of outcome evidence as high, moderate, low, or very low, which can be downgraded for five reasons (risk of bias, imprecision, inconsistency, indirectness, and publication bias) and upgraded for three reasons (large magnitude of an effect, doseresponse gradient, and effect of plausible residual confounding).

\section{RESULTS}

\section{Study Selection}

The search strategy retrieved 6,144 studies: 6,099 were identified using database searches and 45 identified using register searches (Supplementary Table S1). After removing 2,579 duplicates from database sources, we screened the retrieved titles and abstracts and excluded 3,487 studies. Of the remaining 78 studies, 48 were excluded because they did not meet the diagnostic or outcome criteria, were not full-text articles, were duplicate studies, did not clearly specify therapy duration, or contained unclear data (Supplementary Table S2). In October 2021, we supplemented the retrieval of four studies that met the inclusion criteria. Finally, 34 studies (Liu and Liu, 2005; Mo, 2010; 


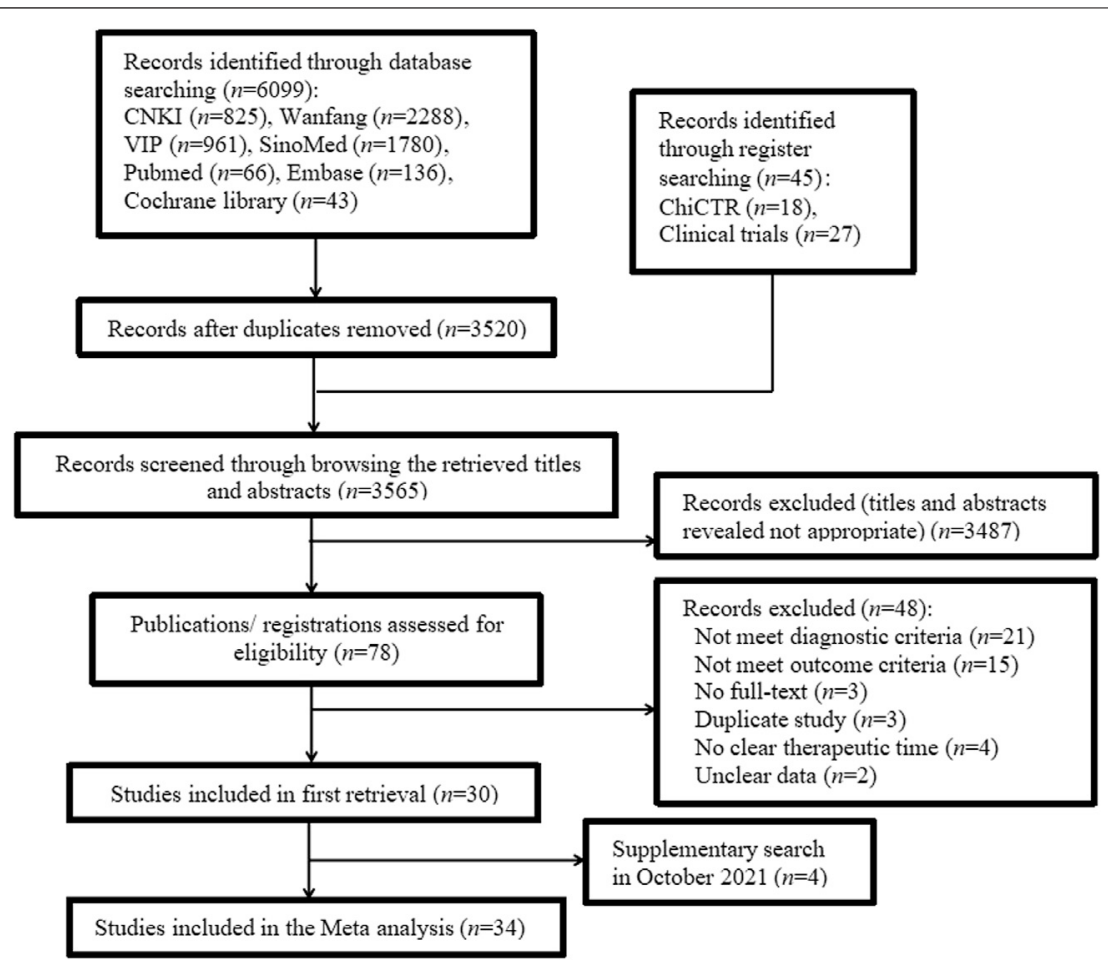

FIGURE 1 | Flowchart of study inclusion.

Cheng et al., 2012; Liu, 2013; Liu, 2014; Xu et al., 2014; Liu, 2015; Luo and Liu, 2016; Ma and Lin, 2016; Tan et al., 2016; Zhuo et al., 2016; Feng et al., 2017; Jiang et al., 2018; Liu, 2018; Ren et al., 2018; Song, 2018; Wang et al., 2018; Huang et al., 2019a; Huang et al., 2019b; Huang, 2019; Liu, 2019; Xu et al., 2019; You, 2019; Wang, 2020b; Chen et al., 2020; Han, 2020; Liu, 2020; Liu et al., 2020; Ma and Zhou, 2020; Meng et al., 2020; Wang et al., 2021b; Li, 2021; Liu and Yuan, 2021; Yang et al., 2021) were included. The screening process is summarized in the flow diagram in Figure 1.

\section{Study Inclusion Characteristics}

We included 34 studies in this review. All studies were published in Chinese and conducted between 2005 and 2021. Of the included studies, four studies (Liu and Liu, 2005; Jiang et al., 2018; Liu, 2019; Meng et al., 2020) provided data on PSD and ten studies (Mo, 2010; Xu et al., 2014; Luo and Liu, 2016; Ren et al., 2018; Wang et al., 2018; You, 2019; Han, 2020; Liu et al., 2020; Ma and Zhou, 2020; Yang et al., 2021) provided data on PSCIND. The sample size of the included trials varied from 40 to 122 participants, aged $45-85$ years. The course of treatment ranged from 0.5 to 6 months. A total of 26 studies used the MMSE as the primary outcome measure and 20 studies used the MoCA. Details of the exposure and outcomes of each included study are summarized in Table $\mathbf{1 .}$

\section{Risk of Bias Assessment}

The overall risk of bias was identified high in sixteen studies (Meng et al., 2020; Yang et al., 2021; Liu et al., 2020; Han, 2020;
Ren et al., 2018; Luo and Liu, 2016; Xu et al., 2014; Mo, 2010; Li, 2021; Liu, 2020; Wang, 2020; Xu et al., 2019; Huang et al., 2019b; Huang et al., 2019a; Huang, 2019; Liu, 2018), moderate in seven studies (Liu and Liu, 2005; Liu and Yuan, 2021; Chen et al., 2020; Liu, 2015; Liu, 2014; Liu, 2013; Cheng et al., 2012) and low in eleven studies (Liu, 2019; Jiang et al., 2018; Ma and Zhou, 2020; You, 2019; Wang et al., 2018; Wang et al., 2021b; Song, 2018; Feng et al., 2017; Ma and Lin, 2016; Tan et al., 2016; Zhuo et al., 2016), as is shown in Figure $\mathbf{2}$ and Supplementary Figure S1. The randomization process was considered some concerns as thirteen studies (Liu and Liu, 2005; Cheng et al., 2012; Liu, 2013; Liu, 2014; Xu et al., 2014; Liu, 2015; Luo and Liu, 2016; Xu et al., 2019; Chen et al., 2020; Liu, 2020; Liu et al., 2020; Li, 2021; Liu and Yuan, 2021) did not provide enough information in the method of random sequence generation. We rated some concerns in the deviations from intended interventions for the use of perprotocol analysis of five studies (Liu, 2018; Huang, 2019; Wang, 2020b; Han, 2020; Liu, 2020), and the missing outcome data of four studies (Liu, 2018; Huang, 2019; Wang, 2020b; Liu, 2020) were showed high risk due to the shedding cases were mostly depend on the clinical efficacy of drugs. The risk of bias in the measurement of the outcome in fourteen studies (Mo, 2010; Xu et al., 2014; Luo and Liu, 2016; Liu, 2018; Ren et al., 2018; Huang et al., 2019a; Huang et al., 2019b; Huang, 2019; Xu et al., 2019; Wang, 2020b; Han, 2020; Meng et al., 2020; Li, 2021; Yang et al., 2021) were evaluated as high because the use of composite index to measure clinical efficacy rate which could not objectively reflect the intervention effect of TCM treatment. 
TABLE 1 | Characteristics of included studies.

\begin{tabular}{|c|c|c|c|c|c|c|c|c|c|c|c|c|}
\hline \multirow[t]{2}{*}{ Study ID } & \multicolumn{2}{|c|}{ Sample size } & \multicolumn{2}{|c|}{ Course of disease (month) } & \multicolumn{2}{|c|}{ Mean age (year) } & \multicolumn{2}{|c|}{ Male/Female (Male\%) } & \multicolumn{2}{|c|}{ Intervention } & \multirow{2}{*}{$\begin{array}{c}\text { Course of } \\
\text { treatment } \\
\text { (month) }\end{array}$} & \multirow[t]{2}{*}{ Outcomes } \\
\hline & Trial & Control & Trial & Control & Trial & Control & Trial & Control & Trial & Control & & \\
\hline $\begin{array}{l}\text { Meng et al. } \\
(2020)\end{array}$ & 42 & 42 & $5.11 \pm 1.27$ & $5.07 \pm 1.35$ & $67.49 \pm 4.93$ & $68.54 \pm 3.64$ & $25 / 17(60 \%)$ & $23 / 19(55 \%)$ & $\begin{array}{l}\text { Peiyuan Tongnao } \\
\text { capsule + } \\
\text { Oxiracetam }\end{array}$ & Oxiracetam & 3 & (2) (4) (5) (7) \\
\hline Liu (2019) & 46 & 46 & $7.21 \pm 1.02$ & $7.15 \pm 0.96$ & $62.95 \pm 2.68$ & $63.02 \pm 2.74$ & 23/23 (50\%) & $21 / 25(46 \%)$ & $\begin{array}{l}\text { Yishen Huayu } \\
\text { Tongluo decoction + } \\
\text { Nimodipine + } \\
\text { Piracetam }\end{array}$ & $\begin{array}{l}\text { Nimodipine + } \\
\text { Piracetam }\end{array}$ & 6 & (2) $(7)$ \\
\hline $\begin{array}{l}\text { Jiang et al., } \\
\text { 2018) }\end{array}$ & 40 & 40 & $2.91 \pm 1.07$ & $2.45 \pm 1.21$ & $66.29 \pm 4.11$ & $65.39 \pm 2.87$ & $21 / 19(53 \%)$ & $23 / 17(58 \%)$ & $\begin{array}{l}\text { Tongnaoling granule } \\
\text { + Donepezil }\end{array}$ & Donepezil & 6 & (2) $(7)$ \\
\hline $\begin{array}{l}\text { Liu and Liu } \\
\text { (2005) }\end{array}$ & 60 & 60 & 7 days -3 & 7 days-3.5 & $55-81$ & $78-58$ & $34 / 26(57 \%)$ & $38 / 22(63 \%)$ & $\begin{array}{l}\text { TCM decoction + } \\
\text { Nimodipine }\end{array}$ & Nimodipine & 1 & (2) \\
\hline $\begin{array}{l}\text { Yang et al. } \\
\text { (2021) }\end{array}$ & 27 & 27 & $0.76 \pm 0.47$ years & $0.69 \pm 0.42$ years & $61.10 \pm 15.83$ & $60.82 \pm 16.10$ & $15 / 12(56 \%)$ & $16 / 11(59 \%)$ & $\begin{array}{l}\text { Xiongqi Xingnao } \\
\text { granule }+ \\
\text { Butylphthalide }\end{array}$ & Butylphthalide & 2 & (1) (2) \\
\hline $\begin{array}{l}\text { Liu et al. } \\
\text { (2020) }\end{array}$ & 30 & 30 & $12.56 \pm 2.23 \mathrm{~h}$ & $12.48 \pm 2.45 \mathrm{~h}$ & $61.12 \pm 6.21$ & $61.35 \pm 6.12$ & $15 / 15(50 \%)$ & $17 / 13(57 \%)$ & $\begin{array}{l}\text { Tongqiao Huoxue } \\
\text { decoction }+ \\
\text { Citicoline }\end{array}$ & Citicoline & 0.5 & (1) (5) (7) \\
\hline $\begin{array}{l}\text { Ma and Zhou } \\
(2020)\end{array}$ & 47 & 47 & NR & $N R$ & $64.2 \pm 12.5$ & $63.8 \pm 12.4$ & $26 / 21(55 \%)$ & $28 / 19(60 \%)$ & $\begin{array}{l}\text { Tongnao Yisui } \\
\text { decoction + } \\
\text { Donepezil }\end{array}$ & Donepezil & 3 & (2) \\
\hline Han (2020) & 41 & 43 & $3.59 \pm 1.48$ & $3.23 \pm 1.27$ & $56.77 \pm 4.67$ & $67.63 \pm 3.86$ & 20/21 (49\%) & $19 / 24(46 \%)$ & $\begin{array}{l}\text { Bushen Yizhi } \\
\text { decoction + } \\
\text { Donepezil }\end{array}$ & Donepezil & 3 & (2) (3) (7) \\
\hline You (2019) & 30 & 30 & $N R$ & $N R$ & $63.47 \pm 8.21$ & $63.60 \pm 7.75$ & $15 / 15(50 \%)$ & $13 / 17(43 \%)$ & $\begin{array}{l}\text { Yiqi Shuxue Tongmai } \\
\text { decoction + } \\
\text { Donepezil }\end{array}$ & Donepezil & 3 & (1) $(7)$ \\
\hline $\begin{array}{l}\text { Ren et al. } \\
\text { (2018) }\end{array}$ & 40 & 40 & $3.73 \pm 0.56$ & $3.82 \pm 0.60$ & $67.02 \pm 5.38$ & $67.26 \pm 5.63$ & 22/18 (55\%) & $24 / 16(60 \%)$ & $\begin{array}{l}\text { Yishen Huayu } \\
\text { decoction + } \\
\text { Donepezil }\end{array}$ & Donepezil & 3 & (1) \\
\hline $\begin{array}{l}\text { Wang et al. } \\
\text { (2018) }\end{array}$ & 30 & 30 & $2.8 \pm 0.6$ & $3.1 \pm 0.5$ & $62.73 \pm 9.40$ & $59.53 \pm 9.90$ & $14 / 16(47 \%)$ & 19/11 (63\%) & $\begin{array}{l}\text { Qilong Yizhi granule } \\
+ \text { Nimodipine }\end{array}$ & Nimodipine & 6 & (2) $(7)$ \\
\hline $\begin{array}{l}\text { Luo and Liu } \\
\text { (2016) }\end{array}$ & 30 & 30 & $2.9 \pm 0.6$ & $3.0 \pm 0.4$ & $60.25 \pm 8.55$ & $62.50 \pm 8.40$ & $17 / 13(57 \%)$ & $14 / 16(47 \%)$ & $\begin{array}{l}\text { Modified Ditan } \\
\text { decoction + } \\
\text { Nimodipine }\end{array}$ & Nimodipine & 3 & (2) (3) (5) (7) \\
\hline $\begin{array}{l}\text { Xu et al. } \\
\text { (2014) }\end{array}$ & 30 & 30 & $3.9 \pm 0.6$ & $4.0 \pm 0.4$ & $67.5 \pm 3.05$ & $68.3 \pm 3.2$ & 19/11 (63\%) & $20 / 10(67 \%)$ & $\begin{array}{l}\text { Qiangli Zengzhi } \\
\text { decoction + } \\
\text { Nimodipine }\end{array}$ & Nimodipine & 3 & (1) (2) (7) \\
\hline Mo (2010), ${ }^{a}$ & 30 & 30 & $N R$ & NR & $69.6 \pm 9.6$ & $72.6 \pm 8.5$ & $21 / 9$ (70\%) & $20 / 10(67 \%)$ & $\begin{array}{l}\text { Ziyin Jiannao tablet + } \\
\text { Nimodipine }\end{array}$ & Nimodipine & 2 & (2) (6) \\
\hline Li (2021) & 44 & 44 & $1.01 \pm 0.38$ years & $1.03 \pm 0.31$ years & $66.57 \pm 4.48$ & $66.14 \pm 4.53$ & 23/21 (52\%) & $24 / 20(55 \%)$ & $\begin{array}{l}\text { TCM decoction + } \\
\text { Nimodipine }\end{array}$ & Nimodipine & 3 & (1) (3) (7) \\
\hline $\begin{array}{l}\text { Liu and Yuan } \\
\text { (2021) }\end{array}$ & 47 & 47 & $14.52 \pm 2.79$ days & $14.35 \pm 2.68$ days & $62.73 \pm 7.06$ & $62.15 \pm 6.79$ & $27 / 20$ (57\%) & $26 / 21(55 \%)$ & $\begin{array}{l}\text { Huoxue Huayu } \\
\text { Xingnao decoction + } \\
\text { Nimodipine }\end{array}$ & Nimodipine & 1 & (2) (5) \\
\hline $\begin{array}{l}\text { Wang et al. } \\
\text { (2021b) }\end{array}$ & 61 & 61 & $19.56 \pm 3.25$ days & $18.98 \pm 4.38$ days & $68.79 \pm 5.42$ & $69.23 \pm 4.79$ & $39 / 22$ (64\%) & $37 / 24(61 \%)$ & $\begin{array}{l}\text { Chaimu Xingnao } \\
\text { decoction }+ \\
\text { Oxiracetam }\end{array}$ & Oxiracetam & 1 & (1) (2) (3) (7) \\
\hline
\end{tabular}


TABLE 1 | (Continued) Characteristics of included studies.

\begin{tabular}{|c|c|c|c|c|c|c|c|c|c|c|c|c|}
\hline \multirow[t]{2}{*}{ Study ID } & \multicolumn{2}{|c|}{ Sample size } & \multicolumn{2}{|c|}{ Course of disease (month) } & \multicolumn{2}{|c|}{ Mean age (year) } & \multicolumn{2}{|c|}{ Male/Female (Male\%) } & \multicolumn{2}{|c|}{ Intervention } & \multirow{2}{*}{$\begin{array}{l}\text { Course of } \\
\text { treatment } \\
\text { (month) }\end{array}$} & \multirow[t]{2}{*}{ Outcomes } \\
\hline & Trial & Control & Trial & Control & Trial & Control & Trial & Control & Trial & Control & & \\
\hline $\begin{array}{l}\text { Chen et al. } \\
\text { (2020) }\end{array}$ & 40 & 40 & $1.28 \pm 1.12$ weeks & $1.29 \pm 1.08$ weeks & $65.52 \pm 3.94$ & $65.59 \pm 3.91$ & 22/18 (55\%) & 23/17 (58\%) & $\begin{array}{l}\text { Buyang Huanwu } \\
\text { decoction }+ \\
\text { Aniracetam }\end{array}$ & Aniracetam & 1 & (1) (2) (7) \\
\hline Liu (2020) & 30 & 30 & NR & NR & $70.03 \pm 8.79$ & $67.77 \pm 10.51$ & $18 / 12(60 \%)$ & $16 / 14(53 \%)$ & $\begin{array}{l}\text { Shenrong Tongmai } \\
\text { capsule + Donepezil }\end{array}$ & Donepezil & 3 & (1) (2) (3) (7) \\
\hline $\begin{array}{l}\text { Wang } \\
\text { (2020b) }\end{array}$ & 37 & 37 & NR & NR & $60.54 \pm 11.99$ & $62.54 \pm 11.49$ & $17 / 20(46 \%)$ & 22/15 (59\%) & $\begin{array}{l}\text { Zhongfeng Xingnao } \\
\text { liquid + Donepezil }\end{array}$ & Donepezil & 1 & (1) (2) (3) (5) (7) \\
\hline $\begin{array}{l}\text { Xu et al. } \\
\text { (2019) }\end{array}$ & 30 & 30 & NR & NR & NR & NR & NR & NR & $\begin{array}{l}\text { Modified Qufeng } \\
\text { Tongqiao decoction } \\
\text { + Donepezil }\end{array}$ & Donepezil & 1.5 & (1) (2) (7) \\
\hline $\begin{array}{l}\text { Huang et al. } \\
\text { (2019b) }\end{array}$ & 41 & 39 & $2.68 \pm 1.75$ years & $3.12 \pm 1.34$ years & $68.50 \pm 4.90$ & $65.18 \pm 5.20$ & 23/18 (56\%) & $21 / 18(54 \%)$ & $\begin{array}{l}\text { Modified Wuzi } \\
\text { Yanzong pill + } \\
\text { Donepezil }\end{array}$ & Donepezil & 4 & (2) (4) (7) \\
\hline $\begin{array}{l}\text { Huang et al. } \\
\text { (2019a) }\end{array}$ & 47 & 47 & NR & $N R$ & $63.56 \pm 2.24$ & $63.21 \pm 2.19$ & 25/22 (53\%) & $26 / 21(55 \%)$ & $\begin{array}{l}\text { Buchang Naoxintong } \\
\text { capsule + Donepezil }\end{array}$ & Donepezil & 3 & (1) (2) (3) (5) (7) \\
\hline $\begin{array}{l}\text { Huang } \\
\text { (2019) }\end{array}$ & 27 & 30 & NR & NR & $\begin{array}{c}71.00 \\
(69.00,74.00)\end{array}$ & $\begin{array}{c}71.50 \\
(69.00,74.00)\end{array}$ & $21 / 6(78 \%)$ & 20/10 (67\%) & $\begin{array}{l}\text { Zuogui decoction + } \\
\text { Donepezil }\end{array}$ & Donepezil & 3 & (1) (2) (7) \\
\hline Liu (2018) & 40 & 40 & NR & NR & $68.35 \pm 9.76$ & $70.35 \pm 9.75$ & 28/12 (70\%) & 25/15 (62\%) & $\begin{array}{l}\text { Tongnao decoction } \\
+ \text { Donepezil }\end{array}$ & Donepezil & 3 & (2) (3) (5) (7) \\
\hline Song (2018) & 30 & 30 & NR & NR & NR & NR & 19/11 (63\%) & 16/14 (53\%) & $\begin{array}{l}\text { Shuxue Tongmai } \\
\text { granule + Donepezil }\end{array}$ & Donepezil & 3 & (1) (2) (3) (6) (7) \\
\hline $\begin{array}{l}\text { Feng et al. } \\
\text { (2017) }\end{array}$ & 50 & 50 & $4.6 \pm 2.5$ years & $4.5 \pm 2.4$ years & $68.2 \pm 11.3$ & $67.8 \pm 10.8$ & $30 / 20(60 \%)$ & $32 / 18$ (64\%) & $\begin{array}{l}\text { Huannao Yicong } \\
\text { decoction + } \\
\text { Piracetam + } \\
\text { Donepezil }\end{array}$ & $\begin{array}{l}\text { Piracetam + } \\
\text { Donepezil }\end{array}$ & 3 & (1) (2) (3) (7) \\
\hline $\begin{array}{l}\text { Ma and Lin, } \\
\text { 2016) }\end{array}$ & 60 & 60 & NR & NR & $56.5 \pm 6.3$ & $57.4 \pm 6.7$ & $39 / 21$ (65\%) & $36 / 24(60 \%)$ & $\begin{array}{l}\text { Dengzhan Shengmai } \\
\text { capsule + Donepezil }\end{array}$ & Donepezil & 3 & (1) (3) (7) \\
\hline $\begin{array}{l}\text { Tan et al. } \\
\text { (2016), }\end{array}$ & 40 & 40 & $N R$ & $N R$ & $60.1 \pm 4.4$ & $59.2 \pm 6.3$ & $21 / 19(53 \%)$ & 23/17 (58\%) & $\begin{array}{l}\text { Shuxue Tongmai } \\
\text { granule + Donepezil }\end{array}$ & Donepezil & 3 & (1) (3) (6) \\
\hline $\begin{array}{l}\text { Zhuo et al. } \\
\text { (2016) }\end{array}$ & 53 & 53 & NR & NR & $69.9 \pm 7.8$ & $70.2 \pm 7.3$ & $27 / 26$ (51\%) & 29/24 (55\%) & $\begin{array}{l}\text { Yangxue Qingnao } \\
\text { granule + } \\
\text { Butylphthalide }\end{array}$ & Butylphthalide & 1 & (1) (2) \\
\hline Liu (2015) & 42 & 42 & NR & NR & $69.9 \pm 7.2$ & $70.5 \pm 7.0$ & 24/18 (57\%) & 22/20 (52\%) & $\begin{array}{l}\text { Buyang Huanwu } \\
\text { decoction + } \\
\text { Butylphthalide }\end{array}$ & Butylphthalide & 0.5 & (1) \\
\hline Liu (2014), ${ }^{c}$ & 50 & 50 & $N R$ & NR & 58.5 & 20.4 & $47 / 53$ & (47\%) & $\begin{array}{l}\text { Tianzhi granule + } \\
\text { Oxiracetam }\end{array}$ & Oxiracetam & 1 & (2) (4) (7) \\
\hline Liu (2013) & 20 & 20 & NR & NR & $70.8 \pm 9.0$ & $69.6 \pm 9.1$ & 14/6 (70\%) & 15/5 (75\%) & $\begin{array}{l}\text { Tongqiao Huoxue } \\
\text { decoction }+ \\
\text { Nimodipine }\end{array}$ & Nimodipine & 1 & (1) \\
\hline $\begin{array}{l}\text { Cheng et al. } \\
(2012){ }^{\mathrm{C}}\end{array}$ & 44 & 40 & NR & NR & $54-80$ & $55-79$ & 26/18 (59\%) & $24 / 16(60 \%)$ & $\begin{array}{l}\text { Tianzhi granule + } \\
\text { Piracetam }\end{array}$ & Piracetam & 1 & (2) (4) (7) \\
\hline
\end{tabular}

Abbreviations: NR, Not Reported; Montreal Cognitive Assessment (MoCA); Mini-Mental State Examination (MMSE); Barthel Index (BI); Activities of Daily Living Scale (ADL); National Institutes of Health Stroke Scale (NIHSS); China Stroke Scale (CSS); Adverse events.

${ }^{a}$ The CSS, evaluation standard was incorrect, and the data were not included in the study.

${ }^{b}$ The CSS, data for the two groups before and after treatment were the same; this was considered incorrect and was not included in the study.

${ }^{\circ} T$ The $A D L$, data were inconsistent with the description, which was not included in the study. 


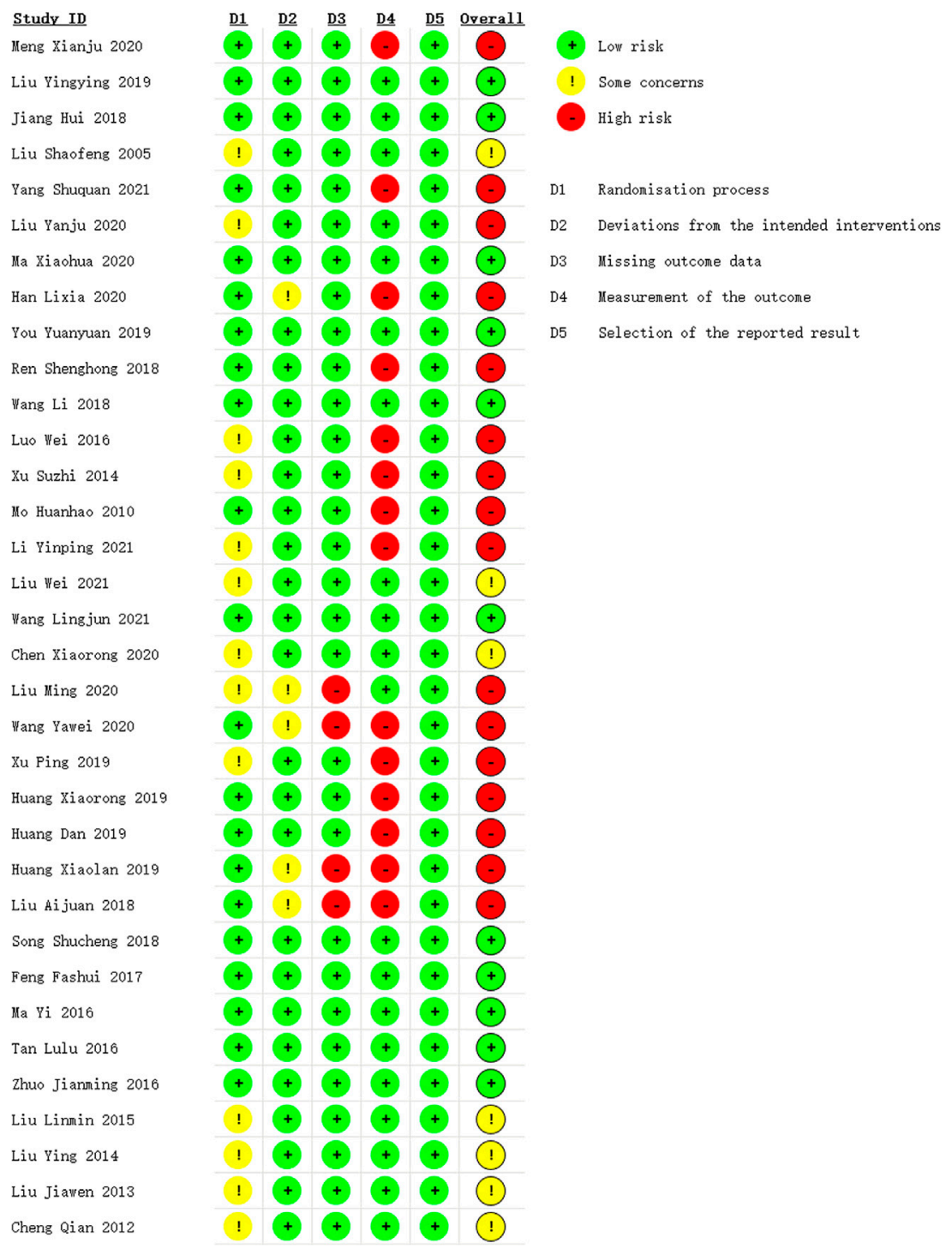

FIGURE 2 | Risk of bias summary.

\section{Cognitive Function Assessment \\ MoCA}

TCM plus conventional Western medication was used in 20 studies, as shown in Figure 3. Of these studies, one study (Chen et al., 2020) lacked information about post-treatment scores, but the 6 months follow-up results showed that TCM adjuvant therapy significantly improved long-term MoCA scores $[\mathrm{MD}=4.14,95 \% \mathrm{CI}(3.19,5.09)]$. The subgroup analysis of therapy duration showed that eight studies (Liu, 2013; Liu, 2015; Zhuo et al., 2016; Xu et al., 2019; Wang, 2020b; Liu et al., 2020; Wang et al., 2021b; Yang et al., 2021) had a therapy duration of $<3$ months $\left(p<0.0001, I^{2}=79 \%\right)$ and eleven studies (Xu et al., 2014; Ma and Lin, 2016; Tan et al., 2016; Feng et al., 2017; Ren et al., 2018; Song, 2018; Huang et al., 2019a; Huang, 2019; You, 2019; Liu, 2020; Li, 2021) had a therapy duration of 3 months ( $p<$ $0.00001, I^{2}=87 \%$ ), suggesting some heterogeneity. The random- effect model showed a significant effect of TCM adjuvant therapy on MoCA scores [MD $=2.55,95 \% \mathrm{CI}(1.56,3.53), p<0.00001$; $\mathrm{MD}=3.07,95 \% \mathrm{CI}(1.98,4.17), p<0.00001]$. The funnel plot was asymmetrical, indicating the existence of publication bias (Supplementary Figure S2).

\section{MMSE}

TCM plus conventional Western medication was used in 26 studies, as shown in Figure 4. Of these, one study (Chen et al., 2020) did not mention post-treatment scores, but the 6 months follow-up results showed that TCM adjuvant therapy had a beneficial effect on long-term MMSE scores $[\mathrm{MD}=5.00$, $95 \%$ CI $(3.77,6.23)]$. Subgroup analysis was carried out to examine differences in therapy duration. Ten studies (Liu and Liu, 2005; Mo, 2010; Cheng et al., 2012; Liu, 2014; Zhuo et al., 2016; Xu et al., 2019; Wang, 2020b; Wang et al., 2021b; Liu and 


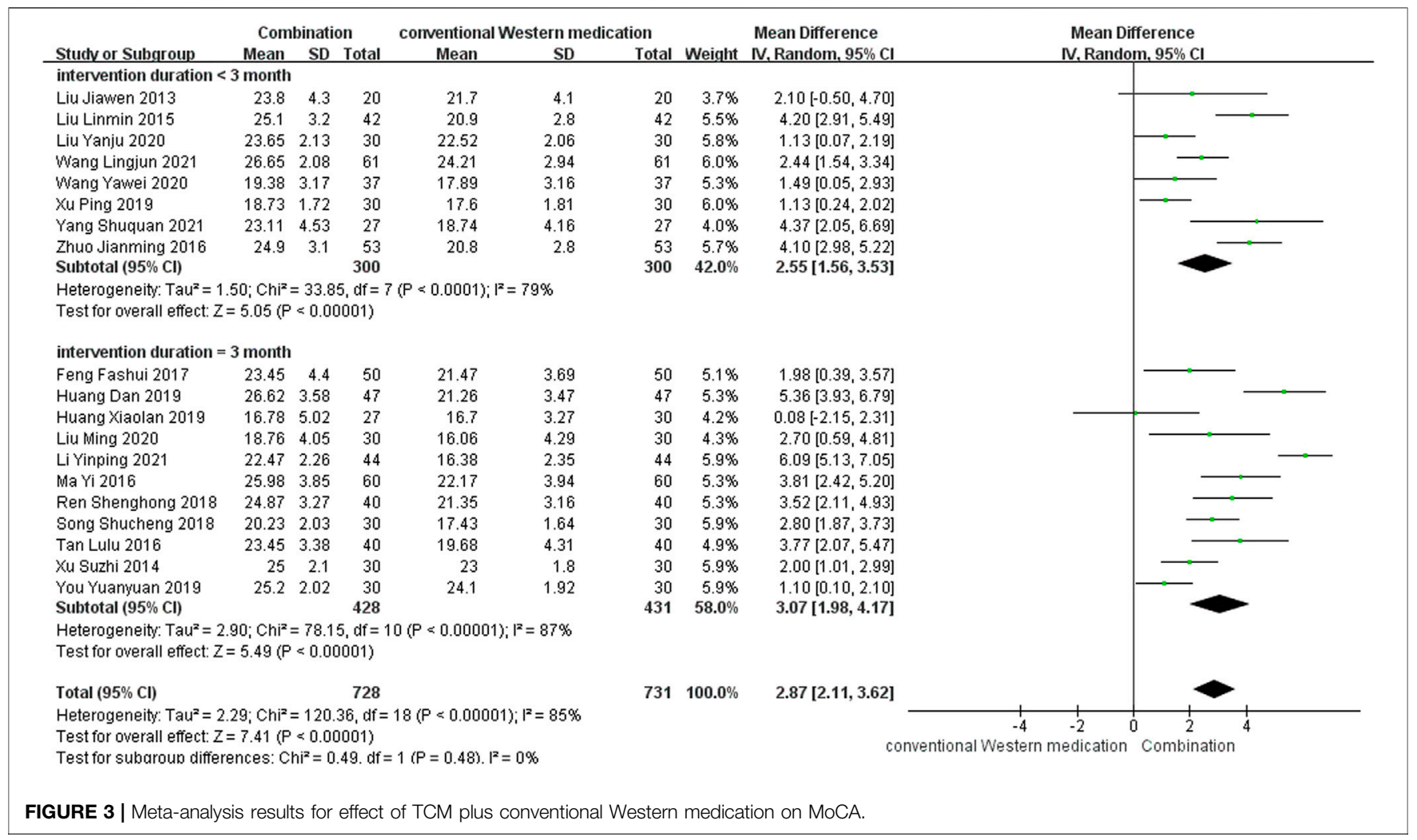

Yuan, 2021; Yang et al., 2021) had a therapy duration of $<3$ months $\left(p=0.03, I^{2}=53 \%\right.$ ), eleven studies (Xu et al., 2014; Luo and Liu, 2016; Feng et al., 2017; Liu, 2018; Song, 2018; Huang et al., 2019a; Huang, 2019; Han, 2020; Liu, 2020; Ma and Zhou, 2020; Meng et al., 2020) had a therapy duration of 3 months ( $p<0.00001, I^{2}=88 \%$ ), one study (Huang et al., 2019b) reported a therapy duration of 4 months, and three studies (Jiang et al., 2018; Wang et al., 2018; Liu, 2019) had a therapy duration of 6 months $\left(p<0.00001, I^{2}=93 \%\right)$, suggesting some heterogeneity. The random-effect model showed a significant beneficial effect of TCM adjuvant therapy on MMSE scores $[\mathrm{MD}=2.55,95 \% \mathrm{CI}$ $(1.99,3.10), p<0.00001 ; \mathrm{MD}=2.53,95 \%$ CI $(1.59,3.47), p<$ $0.00001 ; \mathrm{MD}=2.91,95 \% \mathrm{CI}(1.26,4.56), p=0.0006 ; \mathrm{MD}=3.11$, $95 \%$ CI $(-0.04,6.27), p=0.05]$. The funnel plot was asymmetrical, indicating the existence of publication bias (Supplementary Figure S3).

\section{Activities of Daily Living \\ BI}

TCM plus conventional Western medication was used in 12 studies, as shown in Figure 5. Subgroup analysis was carried out to examine differences in therapy duration. Two study (Wang, 2020b; Wang et al., 2021b) had a therapy duration of $<3$ months $\left(p=0.11, I^{2}=\right.$ 61\%) and ten studies (Luo and Liu, 2016; Ma and Lin, 2016; Tan et al., 2016; Feng et al., 2017; Liu, 2018; Song, 2018; Huang et al., 2019a; Han, 2020; Liu, 2020; Li, 2021) had a therapy duration of 3 months ( $p<0.00001, I^{2}=93 \%$ ), suggesting significant heterogeneity. The random-effect model showed a significant beneficial effect of TCM adjuvant therapy on $\mathrm{BI}$ scores $[\mathrm{MD}=7.34,95 \% \mathrm{CI}(3.83,10.85), p<$
$0.0001 ; \mathrm{MD}=8.98,95 \% \mathrm{CI}(4.76,13.21), p<0.0001]$. The funnel plot was asymmetrical, indicating the existence of publication bias (Supplementary Figure S4).

ADL

TCM plus conventional Western medication was used in two studies, as shown in Table 2 . The therapy duration was 3 months (Meng et al., 2020) and 4 months (Huang X. R. et al., 2019) respectively, and the results suggest a beneficial effect on $\mathrm{ADL}$ scores $[\mathrm{MD}=-8.64,95 \% \mathrm{CI}(-9.83,-7.45), p<0.00001 ; \mathrm{MD}=$ $-2.00,95 \%$ CI $(-2.94,-1.06), p<0.0001]$.

\section{Neurological Deficit NIHSS}

TCM plus conventional Western medication was observed in seven studies, as shown in Figure 6. The subgroup analysis of therapy duration showed that three studies (Wang, 2020b; Liu et al., 2020; Liu and Yuan, 2021) had a therapy duration of $<3$ months ( $p<0.00001$, $I^{2}=97 \%$ ). Four studies (Luo and Liu, 2016; Liu, 2018; Huang D. et al., 2019a; Meng et al., 2020) had a therapy duration of 3 months ( $p<$ $0.00001, I^{2}=95 \%$ ), suggesting significant heterogeneity. The randomeffect model showed that TCM adjuvant therapy significantly improved NIHSS scores [MD $=-2.48,95 \%$ CI $(-4.97,0.00), p=$ $0.05 ; \mathrm{MD}=-3.81,95 \% \mathrm{CI}(-6.21,-1.40), p=0.002]$.

\section{CSS}

TCM plus conventional Western medication was used in one study. The therapy duration was 3 months (Song, 2018). The results suggest a significant effect of TCM adjuvant therapy on CSS scores [MD = $-2.47,95 \%$ CI $(-3.49,-1.45), p<0.00001]$. 


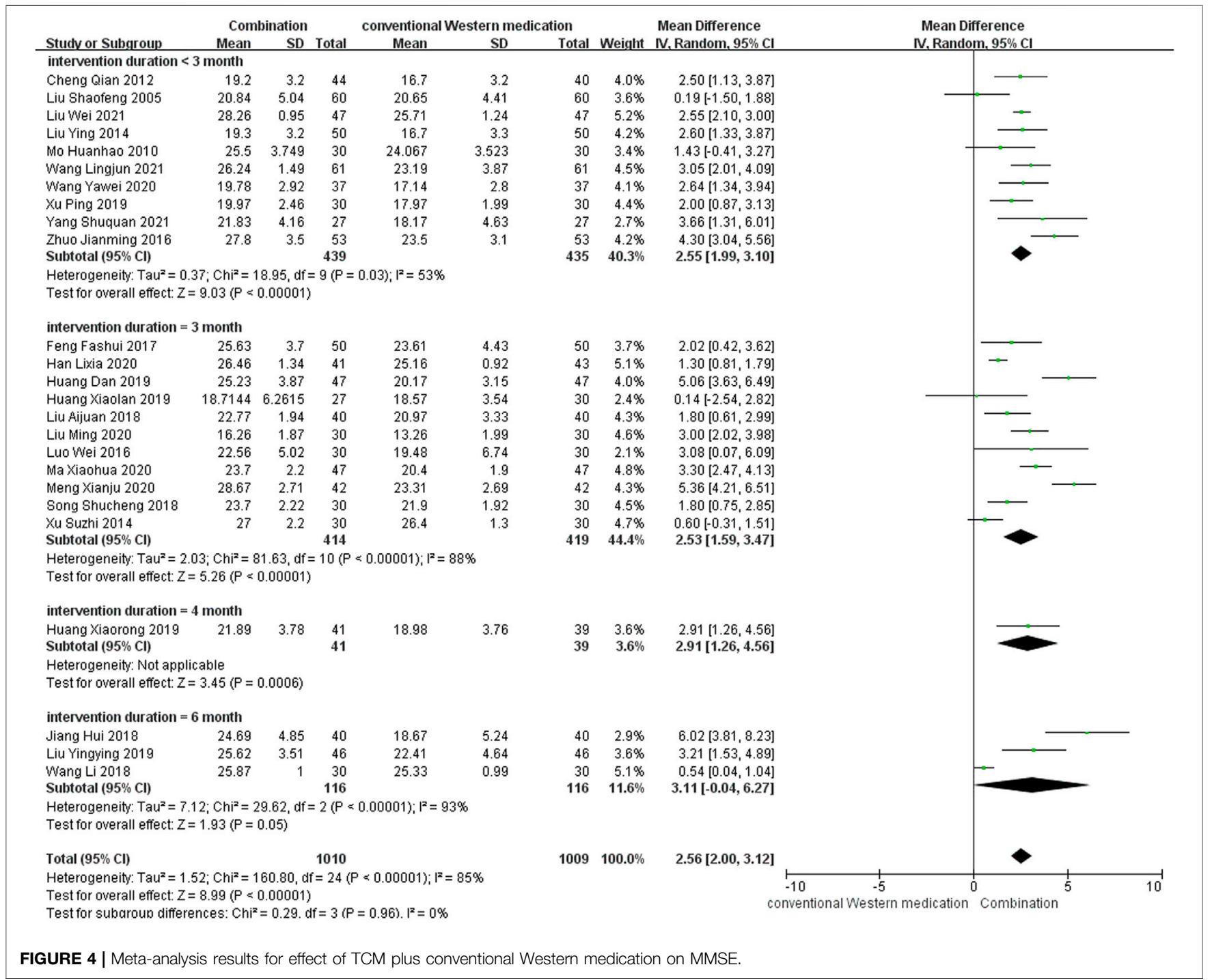

\section{Adverse Events}

A total of 23 studies provided safety-related data. Of these, 11 studies (Cheng et al., 2012; Liu, 2014; Luo and Liu, 2016; Jiang et al., 2018; Wang et al., 2018; Huang et al., 2019b; Huang, 2019; Wang, 2020b; Han, 2020; Liu, 2020; Li, 2021) reported no adverse events, so they were not included in this meta-analysis. A total of 12 studies (Xu et al., 2014; Ma and Lin, 2016; Feng et al., 2017; Song, 2018; Huang et al., 2019a; Liu, 2019; Xu et al., 2019; You, 2019; Chen et al., 2020; Liu et al., 2020; Meng et al., 2020; Wang et al., 2021b) reported gastrointestinal side effects in the intervention group and eight studies (Ma and Lin, 2016; Feng et al., 2017; Huang et al., 2019a; Liu, 2019; Chen et al., 2020; Liu et al., 2020; Meng et al., 2020; Wang et al., 2021b) reported gastrointestinal side effects in the control group, including nausea, vomiting, constipation, stomach discomfort, and loss of appetite. A total of five studies (Ma and Lin, 2016; Feng et al., 2017; Huanget al., 2019a; Chen et al., 2020; Wang et al., 2021b) reported nervous system side effects in the intervention group and six studies (Xu et al., 2014; Huang et al., 2019a; Chen et al., 2020; Liu et al., 2020; Meng et al., 2020; Wang et al., 2021b) reported nervous system side effects in the control group, including drowsiness, insomnia, dizziness, and fatigue. In addition, four studies (Liu, 2019; Chen et al., 2020; Liu et al., 2020; Meng et al., 2020) and six studies (Feng et al., 2017; Song, 2018; Huang et al., 2019a; You, 2019; Chen et al., 2020; Liu et al., 2020) reported dry mouth, frequent urination, rash, blood pressure fluctuations, and other adverse events in the intervention and control groups, respectively. The adverse events were mostly gastrointestinal discomfort, and no serious adverse reactions were found, as shown in Figure 7. The funnel plot was asymmetrical, indicating the existence of publication bias (Supplementary Figure S5).

\section{GRADE Assessment}

GRADE system was used to evaluate the overall evidence of the above six outcomes, as is shown in Table 3. The certainty of evidence indicated very low due to suspected publication bias, significant heterogeneity and serious methodological problems. 


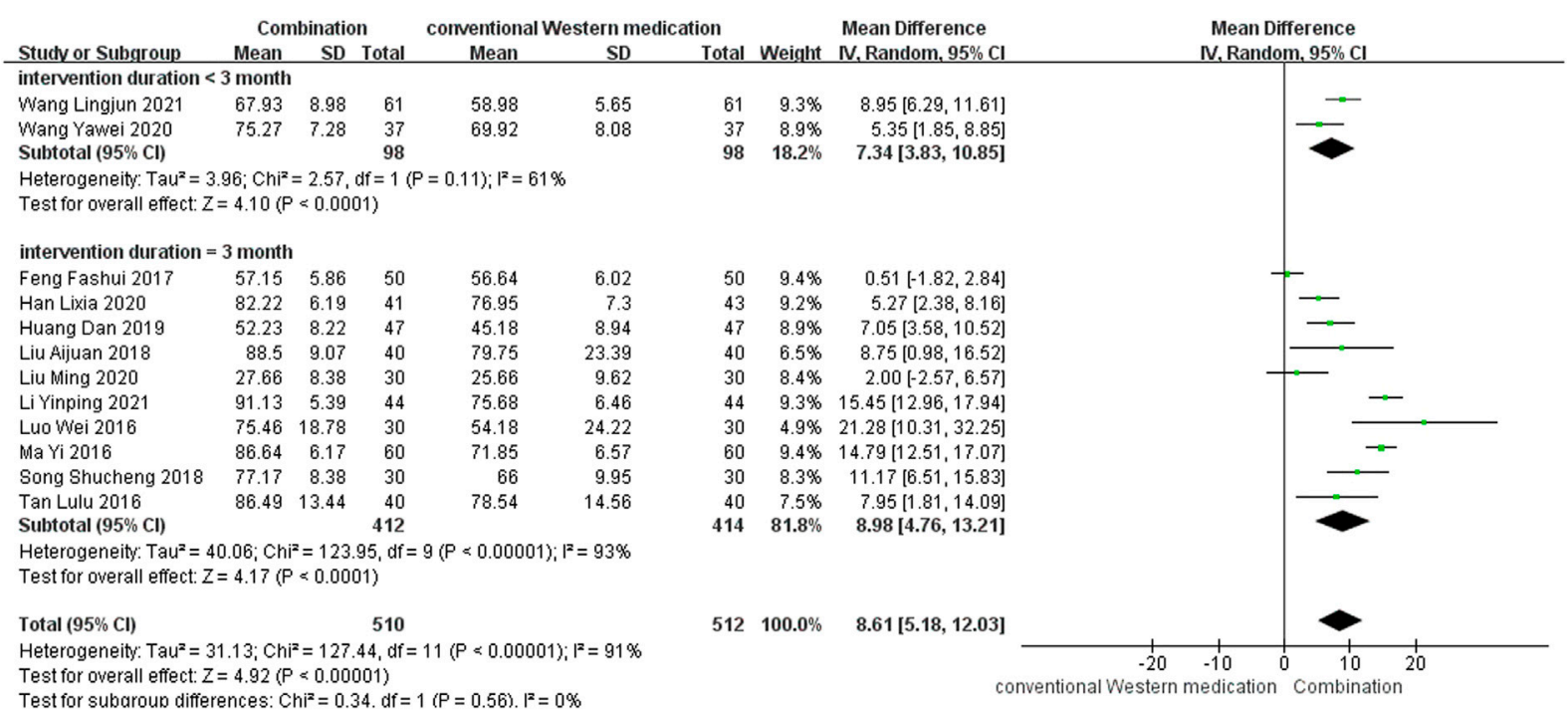

FIGURE 5 | Meta-analysis results for effect of TCM plus conventional Western medication on BI.

TABLE 2 | Meta-analysis results for effect of TCM plus conventional Western medication on ADL.

\begin{tabular}{|c|c|c|c|c|c|c|}
\hline \multirow[t]{2}{*}{ Study ID } & \multirow[t]{2}{*}{ Intervention duration } & \multicolumn{2}{|c|}{ Intervention group } & \multicolumn{2}{|c|}{ Control group } & \multirow[t]{2}{*}{ MD $[95 \% \mathrm{Cl}]$} \\
\hline & & $-x \pm s$ & $n$ & $-x \pm s$ & $n$ & \\
\hline Meng et al. (2020) & $=3$ months & $17.31 \pm 2.51$ & 42 & $25.95 \pm 3.04$ & 42 & $-8.64(-9.83,-7.45)$ \\
\hline Huang et al. (2019b) & $=4$ months & $16 \pm 2.675$ & 41 & $18 \pm 1.459$ & 39 & $-2.00(-2.94,-1.06)$ \\
\hline
\end{tabular}

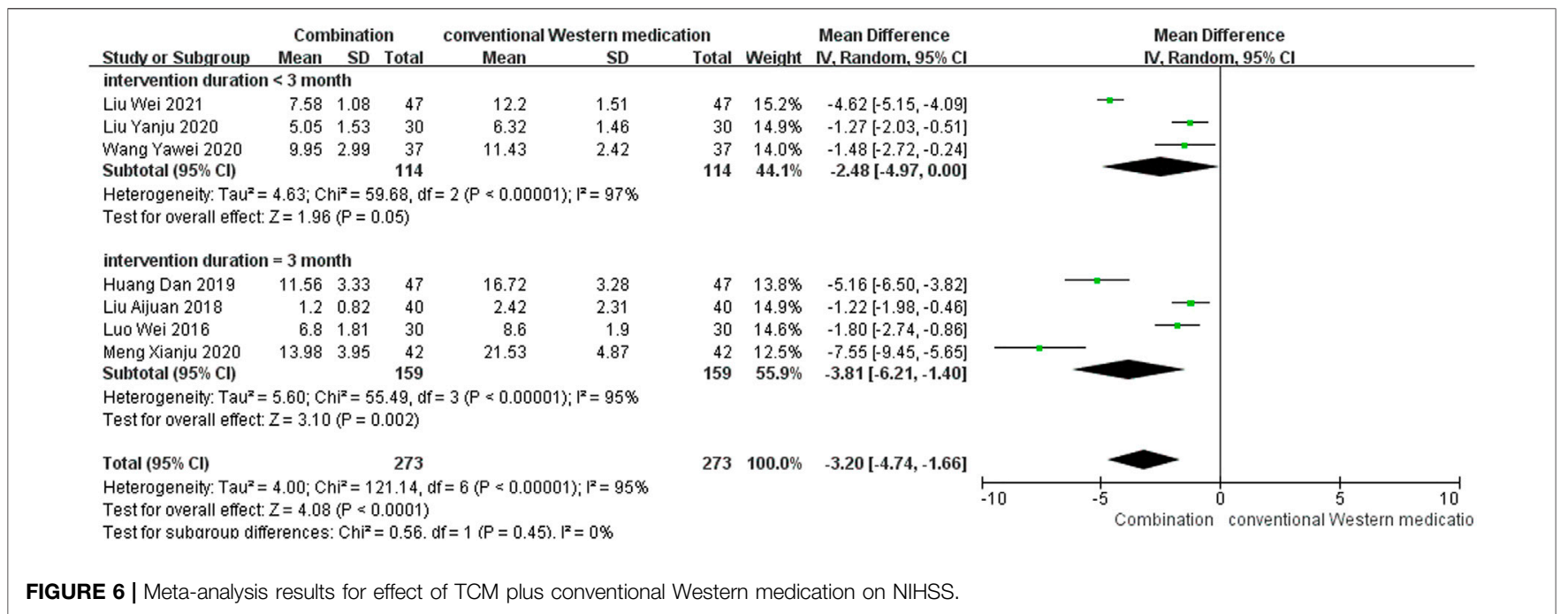

\section{DISCUSSION}

\section{Summary of Evidence}

In this systematic review, we evaluated the clinical efficacy of TCM adjuvant therapy in the treatment of PSCI. A rigorous and reproducible methodology was used to search the literature, and 34 studies involving 2,711 patients with PSCI were identified for analysis. Subgroup analyses of treatment duration showed that TCM adjuvant therapy can promote the recovery of cognitive function, improve activities of daily living, and reduce neurological deficit symptoms in stroke patients. Of the 34 studies analyzed, 11 reported no adverse events related to TCM interventions, and adverse reactions of the remaining studies mainly comprised gastrointestinal discomfort. This 


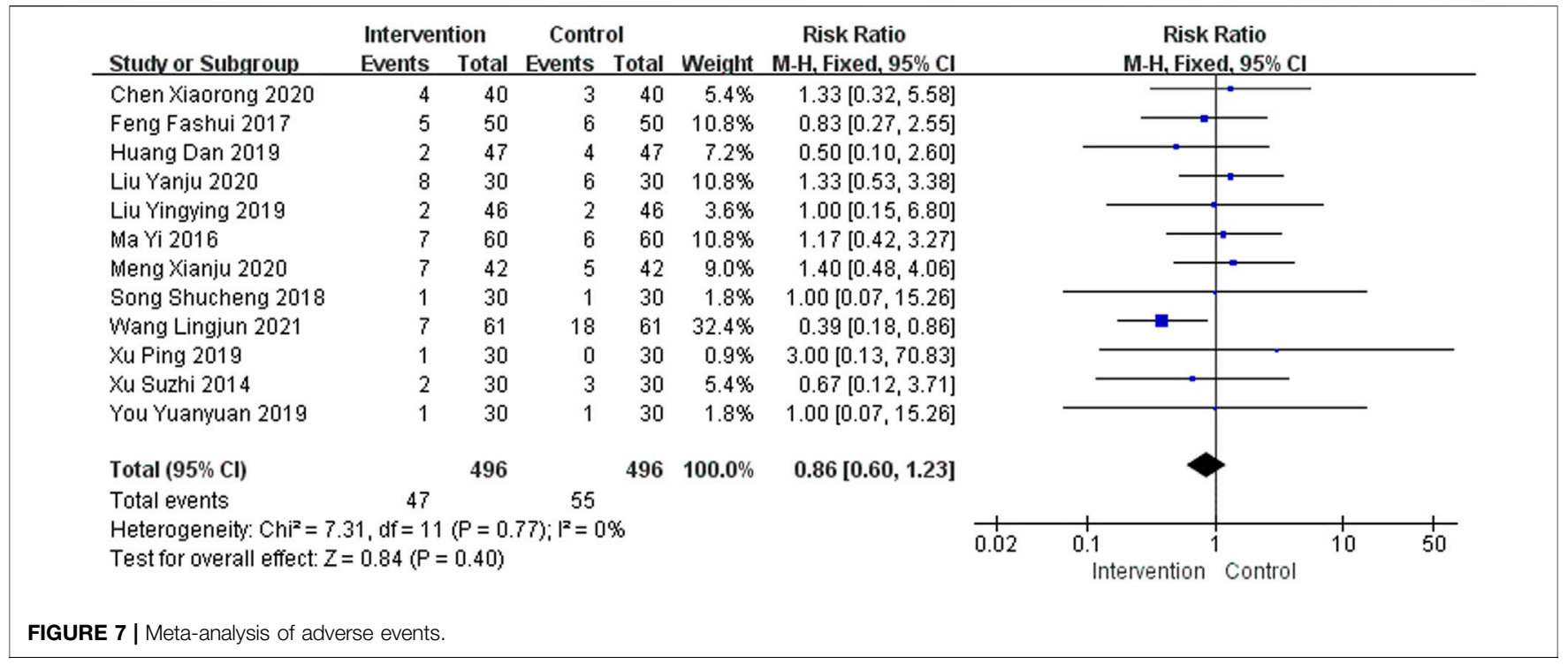

TABLE 3 | Certainty assessment of evidence according to GRADE.

\begin{tabular}{|c|c|c|c|c|c|c|c|c|}
\hline Outcomes & $\begin{array}{l}\text { Risk } \\
\text { of bias }\end{array}$ & Inconsistency & Indirectness & Imprecision & $\begin{array}{l}\text { Publication } \\
\text { bias }\end{array}$ & $\begin{array}{c}\text { No. } \\
\text { of } \\
\text { patients } \\
\text { (studies) }\end{array}$ & $\begin{array}{c}\text { Absolute } \\
\text { effects } \\
(95 \% \\
\text { Cl) }\end{array}$ & $\begin{array}{c}\text { Certainty } \\
\text { of the } \\
\text { evidence }\end{array}$ \\
\hline MoCA & serious $^{a}$ & very serious ${ }^{b}$ & not serious & not serious & $\begin{array}{l}\text { strongly } \\
\text { suspected }^{\mathrm{c}}\end{array}$ & $1,459(19)$ & $\begin{array}{l}\text { MD } 2.87 \text { higher ( } 2.11 \text { higher-3.62 } \\
\text { higher) }\end{array}$ & $\begin{array}{l}\oplus \bigcirc \bigcirc \bigcirc \text { Very } \\
\text { low }\end{array}$ \\
\hline MMSE & serious $^{a}$ & very serious ${ }^{b}$ & not serious & not serious & $\begin{array}{l}\text { strongly } \\
\text { suspected }^{\mathrm{C}}\end{array}$ & $2019(25)$ & $\begin{array}{l}\text { MD } 2.56 \text { higher ( } 2 \text { higher-3.12 } \\
\text { higher) }\end{array}$ & $\begin{array}{l}\oplus \bigcirc \bigcirc \bigcirc \text { Very } \\
\text { low }\end{array}$ \\
\hline $\mathrm{Bl}$ & serious $^{a}$ & very serious ${ }^{b}$ & not serious & not serious & $\begin{array}{l}\text { strongly } \\
\text { suspected }^{c}\end{array}$ & 1,022 (12) & $\begin{array}{l}\text { MD } 8.61 \text { higher ( } 5.18 \text { higher-12.03 } \\
\text { higher) }\end{array}$ & $\begin{array}{l}\oplus \bigcirc \bigcirc \bigcirc \text { Very } \\
\text { low }\end{array}$ \\
\hline ADL & $\begin{array}{l}\text { very } \\
\text { serious }^{d}\end{array}$ & serious $^{e}$ & not serious & not serious & $\begin{array}{l}\text { strongly } \\
\text { suspected }^{f}\end{array}$ & $164(2)$ & $\begin{array}{l}\text { MD } 4.54 \text { lower (5.28 lower-3.8 } \\
\text { lower) }\end{array}$ & $\begin{array}{l}\oplus \bigcirc \bigcirc \bigcirc \text { Very } \\
\text { low }\end{array}$ \\
\hline NIHSS & serious $^{\mathrm{a}}$ & very serious ${ }^{b}$ & not serious & not serious & $\begin{array}{l}\text { strongly } \\
\text { suspected }^{f}\end{array}$ & $546(7)$ & $\begin{array}{l}\text { MD } 3.2 \text { lower ( } 4.74 \text { lower-1.66 } \\
\text { lower) }\end{array}$ & $\begin{array}{l}\oplus \bigcirc \bigcirc \bigcirc \text { Very } \\
\text { low }\end{array}$ \\
\hline CSS & not serious & serious $^{e}$ & not serious & serious $^{g}$ & $\begin{array}{l}\text { strongly } \\
\text { suspected }^{f}\end{array}$ & $60(1)$ & $\begin{array}{l}\text { MD } 2.47 \text { lower (3.49 lower-1.45 } \\
\text { lower) }\end{array}$ & $\begin{array}{l}\oplus \bigcirc \bigcirc \bigcirc \text { Very } \\
\text { low }\end{array}$ \\
\hline
\end{tabular}

Grade assessment with justification given as follows:

${ }^{a}$ Most studies are at high RoB.

$b p^{2} \geq 75 \%$.

${ }^{c}$ Based on the publication bias test, there is apparent asymmetry in the funnel plot.

${ }^{d}$ All studies are at high RoB.

${ }^{\ominus}$ Not possible to determine.

${ }^{\text {f}}$ Too few studies.

${ }^{g}$ Small simple size.

indicates that TCM is generally safe and well tolerated for patients with PSCI, which is consistent with previous findings (Shen et al., 2020). Thus, the findings of this meta-analysis suggest, to a limited extent, that TCM adjuvant therapy can be used for PSCI despite methodological flaws in some studies.

\section{Implications for Practice}

The evidence from this systematic review suggests that the use of TCM as an adjuvant therapy may provide additional benefits to PSCI patients, and is generally safe. Chuanxiong Rhizoma (Chuanxiong), Acorita Tatarinowii Rhizoma (Shichangpu), Pheretima (Dilong), and Angelicae Sinensis Radix (Danggui) were the herbs most frequently used by the retrieved studies and should be considered a core herbal prescription for PSCI that should be investigated in clinical trials.

\section{Implications for Research}

Considering the clinical efficacy of TCM in the treatment of PSCI, we present the following suggestions for future research.

First, scientific design and rational implementation of RCTs are gold standard for evaluating clinical efficacy of interventions (Schulz et al., 2010; Dwan et al., 2019). However, only one study mentioned double-blinding in study design (You, 2019), which result in uncertainty of research conclusions. Therefore, due to 
the very low methodological quality of the included studies, future researchers need to improve the quality of RCTs. Prospective registration of trial protocols (e.g., with the Chinese Clinical Trials Registry), adherence to the CONSORT Extension for Chinese Herbal Medicine Formulas 2017 (Cheng et al., 2017) in reporting the results of RCTs of herbal interventions, and use best practices for designing trials of TCMs (e.g., the SPIRIT-TCM Extension 2018 guidelines (Dai et al., 2019) for standardized design) are highly desirable.

Second, some researchers have used ADAS-cog scores to assess improvements in vascular cognitive impairment patients following various treatments (Chan et al., 2018; Kim et al., 2020b). Additionally, some studies have used this scale to estimate cognitive dysfunction after stroke (Falck et al., 2019). However, this scale does not assess vascular factors associated with executive functions, attention, and mental speed (Price et al., 2005; Jokinen et al., 2006). VaDAS-cog is a revised revision of ADAS-cog that includes five additional subtests reflecting vascular conditions (Bastos et al., 2006). VaDAS-cog may be a more sensitive tool with which to assess patients with vascular burden of the brain (Shi et al., 2020). Therefore, the VaDAS-cog or other appropriate outcome measures should be used in future clinical trials to comprehensively evaluate the cognitive status of patients.

Third, PSCI often impairs everyday activities and affects neurological recovery (Hoffmann et al., 2011). Previous studies indicate that PSCI may be accompanied by obvious mental disorders (Zhong et al., 2021), and poor mental status may be associated with higher mortality (Robinson and Jorge, 2016) and affect cognitive recovery (Medeiros et al., 2020). Therefore, more attention needs to be paid to psychological symptoms as well as cognitive function after stroke. However, only one study (Tan et al., 2016) provided Hamilton Rating Scale for Depression scores before and after treatment. More attention should be paid to the psychological status of patients with PSCI. Psychological status in such patients could be evaluated using the Hamilton Rating Scale for Depression, the Hamilton Rating Scale for Anxiety, or other scales commonly used in clinical practice.

Fourth, there is evidence that cognitive function impairment is persistent and dynamic, and has an accelerated downward trend over 6 years after stroke (Levine et al., 2015). In addition, as some patients experienced cognitive decline shortly after stroke and recovered in subsequent few weeks (Nijsse et al., 2017), PSCI can be divided into early-onset PSCI and delayed-onset PSCI, according to the onset time of cognitive impairment (Mok et al., 2017). However, most of the included studies provided insufficient information on the course of disease, the clinical efficacy of TCM could not be objectively evaluated. Therefore, segmental assessment and follow-up of cognitive function should be carried out to determine the best course and the long-term efficacy of TCM in the treatment of PSCI.

Finally, we ranked the frequency of herbal use in the included studies, and summarized the main active components and possible mechanisms for the most common herbs, which is shown in Table 4. The most frequently used herb for PSCI was Chuanxiong Rhizoma. Tetramethylpyrazine is the main component of this herb. In terms of neuroprotection, tetramethylpyrazine reduces cognitive impairment by regulating the Janus kinase-signal transducer and activator of transcription signaling pathway, while simultaneously reducing brain edema and blood-brain barrier permeability (Chang et al., 2007; Gong et al., 2019; Huang et al., 2021). The second most frequently used herb was Acorita Tatarinowii Rhizoma. The major active ingredients of this herb are $\alpha$-asarone and $\beta$ asarone. These ingredients enhance the proliferation of aberrant neural progenitor cells cultured in vitro and their role in neurodegenerative diseases may be mediated by an increase of expression and secretion of neurotrophic factors in astrocytes (Mao et al., 2015; Lam et al., 2019). Antithrombotic protein and enzymes are the most common components of Pheretima. The extract of Pheretima has excellent anticoagulant and thrombolytic properties, which contribute to the prevention and symptomatic relief of cognitive dysfunction in old age (Ren et al., 2006; Wu et al., 2020). Ligustilide is the main component of Angelicae Sinensis Radix. It may improve cognitive dysfunction by reducing mitochondrial dysfunction, generating an antioxidation effect, and restoring synaptic structure (Xin et al., 2013; Li et al., 2015; $\mathrm{Xu}$ et al., 2018). As the active components in most TCM formulations and the underlying mechanisms of action remain unclear. More detailed mechanistic studies using modern scientific methods and approaches are needed to elucidate the therapeutic potential mechanisms of TCM for PSCI. Welldesigned animal studies and RCTs are also required to validate the physiological and pathological effects of these agents in the treatment of patients with PSCI.

\section{Limitations}

Some limitations were identified in the primary trials, which need to be addressed in future studies. First, the methodological quality of the included studies was generally low. Although we used a rigorous method to retrieve and select literature, publication bias was inevitable because all the eligible studies were Chinese. Additionally, most studies provided insufficient information on blinding, and 13 of the 34 studies did not provide detailed descriptions of randomization methods, which reduces the credibility of the evidence reported by these studies. Besides, most studies did not use sample size estimation, which undoubtedly increased the risk of exaggerating the intervention effect. Second, the included studies showed clinical heterogeneity. Syndrome differentiation is a unique aspect of TCM (Mei, 2011) and regulates TCM treatment of PSCI (Yao, 2020). However, because of the different symptoms of patients, a large variety of TCMs were used as intervention methods in the included studies, with substantial variation in medication composition, dosage, and treatment duration; this may have led to differences between clinical prescriptions. Third, the use of cognitive evaluation scales needs to be discussed. Most included studies used the MoCA and the MMSE to evaluate the cognitive function of patients. Although the MoCA and MMSE are screening tools (Shi et al., 2018), the PSCI detection rate when using these scales is often lower than the actual incidence (Jokinen et al., 2015). Fourth, treatment duration was not standard across studies. Although most studies assumed an 
TABLE 4 | Frequently used herbs and potential mechanisms.

\begin{tabular}{|c|c|c|c|c|}
\hline Herbs & Main components & Beneficial effects & Potential mechanisms & $\begin{array}{l}\text { Experimental } \\
\text { models used }\end{array}$ \\
\hline $\begin{array}{l}\text { Chuanxiong Rhizoma } \\
\text { (Chuanxiong) }\end{array}$ & Tetramethylpyrazine & $\begin{array}{l}\text { (2) decrease the brain edema and blood- } \\
\text { brain barrier permeability }\end{array}$ & $\begin{array}{l}\text { (1) regulate the JAK/STAT signaling } \\
\text { pathway } \\
\text { (2) increase the expression of tight junction } \\
\text { proteins } \\
\text { (3) inhibition of HIF-1alpha and TNF-alpha } \\
\text { activations } \\
\text { (4) inhibition of apoptosis formation }\end{array}$ & Rats \\
\hline $\begin{array}{l}\text { Acorita Tatarinowii } \\
\text { Rhizoma (Shichangpu) }\end{array}$ & $\alpha$-asarone and $\beta$-asarone & $\begin{array}{l}\text { (1) promote neurogenesis } \\
\text { (2) against neurodegeneration and } \\
\text { neurodegenerative disorders }\end{array}$ & $\begin{array}{l}\text { (1) enhance the proliferation of Aberrant } \\
\text { neural progenitor cells cultured in vitro } \\
\text { (2) stimulate the expression and secretion of } \\
\text { neurotrophic factors in astrocytes } \\
\text { (3) regulate the PKA signaling pathway }\end{array}$ & Rats \\
\hline Pheretima (Dilong) & $\begin{array}{l}\text { Antithrombotic protein and } \\
\text { enzymes }\end{array}$ & $\begin{array}{l}\text { (1) prevention or symptomatic relief of } \\
\text { cognitive dysfunction } \\
\text { (2) anticoagulant and thrombolytic } \\
\text { activity }\end{array}$ & $\begin{array}{l}\text { (1) prolong APTT and decrease fibrinogen } \\
\text { content }\end{array}$ & $\begin{array}{l}\text { Pheretima guillelmi; } \\
\text { Rats }\end{array}$ \\
\hline $\begin{array}{l}\text { Angelicae Sinensis Radix } \\
\text { (Danggui) }\end{array}$ & Ligustilide & (1) ameliorate cognitive dysfunction & $\begin{array}{l}\text { (1) alleviate mitochondrial dysfunction } \\
\text { (2) antioxidation effect } \\
\text { (3) restore the synaptic structure }\end{array}$ & Rats \\
\hline
\end{tabular}

JAKISTAT, Janus kinase-signal transducer and activator of transcription; HIF-1alpha, hypoxia-inducible alpha; TNF-alpha, tumor necrosis factor-alpha; PKA, protein kinase A; APTT, activated partial thromboplastin time.

optimum PSCI treatment duration of 6 months (Wang et al., 2021a), most studies used a treatment duration of 0.5-6 months; only three studies had a treatment duration of 6 months. In addition, most eligible studies lacked follow-up information to determine the long-term efficacy of TCM in treating PSCI.

\section{CONCLUSION}

Despite the significant positive results, the present evidence supports, to a limited extent because of the methodological flaws and herbal heterogeneity, that TCM adjuvant therapy can be used for patients with PSCI. While, further rigorous RCTs are warranted to confirm the efficacy and safety of TCM.

\section{DATA AVAILABILITY STATEMENT}

The original contributions presented in the study are included in the article/Supplementary Material, further inquiries can be directed to the corresponding author.

\section{AUTHOR CONTRIBUTIONS}

WS and XF conceived and designed this study. WS and XF retrieved literature, analyzed data, and drafted the manuscript. XF and LW selected studies, extracted data, and assessed the risk of bias. YZ directed the research. All authors approved the final manuscript.

\section{FUNDING}

This research was supported by the National TCM Leading Personnel Support Program (NATCM Personnel and Education Department (2018)) (No. 12), the Innovation Team and Talents Cultivation Program of National Administration of Traditional Chinese Medicine (No. ZYYCXTD-C-202007) and the China Academy of Chinese Medical Sciences Innovation Fund (No. CI2021A01310).

\section{ACKNOWLEDGMENTS}

We would like to express our appreciation to all authors of the primary studies included in the current systematic review. We thank Diane Williams, PhD, from Liwen Bianji (Edanz) (www. liwenbianji.cn), for editing the English text of a draft of this manuscript.

\section{SUPPLEMENTARY MATERIAL}

The Supplementary Material for this article can be found online at: https:/www.frontiersin.org/articles/10.3389/fphar.2022.816333/ full\#supplementary-material 


\section{REFERENCES}

Aam, S., Einstad, M. S., Munthe-Kaas, R., Lydersen, S., Ihle-Hansen, H., Knapskog, A. B., et al. (2020). Post-stroke Cognitive Impairment-Impact of Follow-Up Time and Stroke Subtype on Severity and Cognitive Profile: The Nor-COAST Study. Front. Neurol. 11, 699. doi:10.3389/fneur.2020.00699

Bastos Leite, A. J., van der Flier, W. M., van Straaten, E. C., Scheltens, P., and Barkhof, F. (2006). Infratentorial Abnormalities in Vascular Dementia. Stroke 37 (1), 105-110. doi:10.1161/01.STR.0000196984.90718.8a

Black, C. M., Ritchie, C. W., Khandker, R. K., Wood, R., Jones, E., Hu, X., et al. (2018). Non-professional Caregiver burden Is Associated with the Severity of Patients' Cognitive Impairment. PLoS One 13 (12), e0204110. doi:10.1371/ journal.pone.0204110

Blomgren, C., Samuelsson, H., Blomstrand, C., Jern, C., Jood, K., and Claesson, L. (2019). Long-term Performance of Instrumental Activities of Daily Living in Young and Middle-Aged Stroke Survivors-Impact of Cognitive Dysfunction, Emotional Problems and Fatigue. PLoS One 14 (5), e0216822. doi:10.1371/ journal.pone. 0216822

Brainin, M., Tuomilehto, J., Heiss, W. D., Bornstein, N. M., Bath, P. M., Teuschl, Y., et al. (2015). Post-stroke Cognitive Decline: an Update and Perspectives for Clinical Research. Eur. J. Neurol. 22 (2), 229e13-386. doi:10.1111/ene.12626

Cai, X., Chen, Z., Pan, X., Xia, L., Chen, P., Yang, Y., et al. (2014). Inhibition of Angiogenesis, Fibrosis and Thrombosis by Tetramethylpyrazine: Mechanisms Contributing to the SDF-1/CXCR4 axis. PLoS One 9 (2), e88176. doi:10.1371/ journal.pone.0088176

Chan, E. S., Bautista, D. T., Zhu, Y., You, Y., Long, J. T., Li, W., et al. (2018). Traditional Chinese Herbal Medicine for Vascular Dementia. Cochrane Database Syst. Rev. 12 (12), CD010284. doi:10.1002/14651858.CD010284.pub2

Chang, Y., Hsiao, G., Chen, S. H., Chen, Y. C., Lin, J. H., Lin, K. H., et al. (2007). Tetramethylpyrazine Suppresses HIF-1alpha, TNF-Alpha, and Activated Caspase-3 Expression in Middle Cerebral Artery Occlusion-Induced Brain Ischemia in Rats. Acta Pharmacol. Sin. 28 (3), 327-333. doi:10.1111/j.17457254.2007.00514.x

Chen, X. R., Wang, M. X., Liu, L., Yu, M., Xu, L., Zhang, Y. W., et al. (2020). Analysis of the Effects of Yiqi Huoxue Method Combined with Aniracetam Capsule in the Treatment of Cognitive Impairment after Stroke and the Changes of Serum NSE and Hs-CRP Levels. J. Liaoning Univ. TCM 22 (12), 14-17. doi:10.13194/j.issn.1673-842x.2020.12.004

Cheng, C. W., Wu, T. X., Shang, H. C., Li, Y. P., Altman, D. G., Moher, D., et al. (2017). CONSORT Extension for Chinese Herbal Medicine Formulas 2017: Recommendations, Explanation, and Elaboration (Traditional Chinese Version). Ann. Intern. Med. 167 (2), W7-W121. doi:10.7326/M16-297710. 7326/IsTranslatedFrom_M17-2977_1

Cheng, Q., Wu, X. Y., and Jing, Y. T. (2012). Effect of Tianzhi Granule Combined with Piracetam on Cognitive Impairment after Cerebral Infarction. Chin. J. Pract. Nervous Dis. 15 (21), 35-37. doi:10.3969/j.issn.1673-5110.2012. 21.019

Cui, G., Shan, L., Guo, L., Chu, I. K., Li, G., Quan, Q., et al. (2015). Novel Antithrombotic Agent for Modulation of Protein Disulfide Isomerase Family Member ERp57 for Prophylactic Therapy. Sci. Rep. 5, 10353. doi:10.1038/ srep10353

Dai, L., Cheng, C. W., Tian, R., Zhong, L. L., Li, Y. P., Lyu, A. P., et al. (2019). Standard Protocol Items for Clinical Trials with Traditional Chinese Medicine 2018: Recommendations, Explanation and Elaboration (SPIRIT-TCM Extension 2018). Chin. J. Integr. Med. 25 (1), 71-79. doi:10.1007/s11655-018-2999-x

Dong, Q., Guo, Q. H., Luo, B. Y., and Xu, Y. (2017). Expert Consensus on the Management of Post-Stroke Cognitive Impairment. Chin. J. Stroke 12 (6), 519-531. doi:10.3969/j.issn.1673-5765.2017.06.011

Dwan, K., Li, T., Altman, D. G., and Elbourne, D. (2019). CONSORT 2010 Statement: Extension to Randomised Crossover Trials. BMJ 366, 14378. doi:10.1136/bmj.14378

Falck, R. S., Best, J. R., Davis, J. C., Eng, J. J., Middleton, L. E., Hall, P. A., et al. (2019). Sleep and Cognitive Function in Chronic Stroke: a Comparative CrossSectional Study. Sleep 42 (5), zsz040. doi:10.1093/sleep/zsz040

Feng, F. S., Wang, J. F., Yu, J. X., and Zhang, J. T. (2017). Effect of Huannao Yicong Decoction on Improving Cognitive Dysfunction after Stroke. Chin. J. Gerontol. 37 (18), 4510-4513. doi:10.3969/j.issn.1005-9202.2017.18.034
Feng, S. T., Wang, X. L., Wang, Y. T., Yuan, Y. H., Li, Z. P., Chen, N. H., et al. (2021). Efficacy of Traditional Chinese Medicine Combined with Selective Serotonin Reuptake Inhibitors on the Treatment for Parkinson's Disease with Depression: A Systematic Review and Meta-Analysis. Am. J. Chin. Med. 49 (3), 627-643. doi:10.1142/S0192415X21500282

Gittler, M., and Davis, A. M. (2018). Guidelines for Adult Stroke Rehabilitation and Recovery. JAMA 319 (8), 820-821. doi:10.1001/jama.2017.22036

Gong, P., Zhang, Z., Zou, Y., Tian, Q., Han, S., Xu, Z., et al. (2019). Tetramethylpyrazine Attenuates Blood-Brain Barrier Disruption in Ischemia/reperfusion Injury through the JAK/STAT Signaling Pathway. Eur. J. Pharmacol. 854, 289-297. doi:10.1016/j.ejphar.2019.04.028

Guyatt, G. H., Oxman, A. D., Vist, G. E., Kunz, R., Falck-Ytter, Y., Alonso-Coello, P., et al. (2008). GRADE: an Emerging Consensus on Rating Quality of Evidence and Strength of Recommendations. BMJ 336 (7650), 924-926. doi:10.1136/bmj.39489.470347.AD

Han, L. X. (2020). Bushen Yizhi Decoction for MCI after Stroke Clinical Observation of Kidney Deficiency Syndrome. Shanxi, China: Shanxi University of Chinese Medicine.

Hoffmann, T., Bennett, S., Koh, C., and Mckenna, K. (2011). The Cochrane Review of Occupational Therapy for Cognitive Impairment in Stroke Patients. Eur. J. Phys. Rehabil. Med. 47 (3), 513-519. doi:10.1002/14651858.CD006430.pub2

Huang, D., Huang, Y. L., Zhao, Z. Y., and Lyu, Y. (2019a). Clinical Efficacy of Donepezil Combined with Buchang Naoxintong Capsule in Treatment of Cognitive Impairment after Cerebral Infarction. Chin. Arch. Traditional Chin. Med. 37 (2), 495-498. doi:10.13193/j.issn.1673-7717.2019.02.060

Huang, X., Yang, J., Huang, X., Zhang, Z., Liu, J., Zou, L., et al. (2021). Tetramethylpyrazine Improves Cognitive Impairment and Modifies the Hippocampal Proteome in Two Mouse Models of Alzheimer's Disease. Front Cel Dev Biol 9, 632843. doi:10.3389/fcell.2021.632843

Huang, X. L. (2019). The Clinical Effect Observation of Modified Zuogui Yin on Patients with Kidney-Essence Deficiency PSCI with Hyperhomocysteinemia. Fujian, China: Fujian University of Traditional Chinese Medicine.

Huang, X. R., Zzhou, S. H., and Si, W. (2019b). Clinical Study of Integrated Traditional Chinese and Western Medicine in Treating 41 Cases of Post-Stroke Cognitive Impairment. Jiangsu J. Traditional Chin. Med. 51 (6), 34-36. doi:10. 3969/j.issn.1672-397X.2019.06.012

Jia, L., Liu, J., Song, Z., Pan, X., Chen, L., Cui, X., et al. (2012). Berberine Suppresses Amyloid-Beta-Induced Inflammatory Response in Microglia by Inhibiting Nuclear Factor-kappaB and Mitogen-Activated Protein Kinase Signalling Pathways. J. Pharm. Pharmacol. 64 (10), 1510-1521. doi:10.1111/j.20427158.2012.01529.x

Jiang, H., Ni, J., and Zheng, Y. (2018). Tongnaoling Granules Combined with Donepezil in the Treatment of post-stroke Cognitive Impairment. Chin. J. Clin. Res. 31 (11), 1582-1585. doi:10.13429/j.cnki.cjcr.2018.11.037

Jokinen, H., Kalska, H., Mäntylä, R., Pohjasvaara, T., Ylikoski, R., Hietanen, M., et al. (2006). Cognitive Profile of Subcortical Ischaemic Vascular Disease. J. Neurol. Neurosurg. Psychiatry 77 (1), 28-33. doi:10.1136/jnnp.2005.069120

Jokinen, H., Melkas, S., Ylikoski, R., Pohjasvaara, T., Kaste, M., Erkinjuntti, T., et al. (2015). Post-stroke Cognitive Impairment Is Common Even after Successful Clinical Recovery. Eur. J. Neurol. 22 (9), 1288-1294. doi:10.1111/ene.12743

Kim, H., Seo, J. S., Lee, S. Y., Ha, K. T., Choi, B. T., Shin, Y. I., et al. (2020a). AIM2 Inflammasome Contributes to Brain Injury and Chronic post-stroke Cognitive Impairment in Mice. Brain Behav. Immun. 87, 765-776. doi:10.1016/j.bbi.2020. 03.011

Kim, J. O., Lee, S. J., and Pyo, J. S. (2020b). Effect of Acetylcholinesterase Inhibitors on post-stroke Cognitive Impairment and Vascular Dementia: A MetaAnalysis. PLoS One 15 (2), e0227820. doi:10.1371/journal.pone.0227820

Kim, M. S., Bang, J. H., Lee, J., Kim, H. W., Sung, S. H., Han, J. S., et al. (2015). Salvia Miltiorrhiza Extract Protects white Matter and the hippocampus from Damage Induced by Chronic Cerebral Hypoperfusion in Rats. BMC Complement. Altern. Med. 15, 415. doi:10.1186/s12906-015-0943-6

Lam, K. Y. C., Wu, Q. Y., Hu, W. H., Yao, P., Wang, H. Y., Dong, T. T. X., et al. (2019). Asarones from Acori Tatarinowii Rhizoma Stimulate Expression and Secretion of Neurotrophic Factors in Cultured Astrocytes. Neurosci. Lett. 707, 134308. doi:10.1016/j.neulet.2019.134308

Lattanzi, S., Coccia, M., Pulcini, A., Cagnetti, C., Galli, F. L., Villani, L., et al. (2020). Endovascular Treatment and Cognitive Outcome after Anterior Circulation Ischemic Stroke. Sci. Rep. 10 (1), 18524. doi:10.1038/s41598-020-75609-1 
Levine, D. A., Galecki, A. T., Langa, K. M., Unverzagt, F. W., Kabeto, M. U., Giordani, B., et al. (2015). Trajectory of Cognitive Decline after Incident Stroke. JAMA 314 (1), 41-51. doi:10.1001/jama.2015.6968

Li, J. J., Zhu, Q., Lu, Y. P., Zhao, P., Feng, Z. B., Qian, Z. M., et al. (2015). Ligustilide Prevents Cognitive Impairment and Attenuates Neurotoxicity in D-Galactose Induced Aging Mice Brain. Brain Res. 1595, 19-28. doi:10.1016/j.brainres.2014. 10.012

Li, Y. P. (2021). Effect of Integrated Traditional Chinese and Western Medicine on Vascular Cognitive Impairment after Cerebral Infarction. J. Pract. Traditional Chin. Med. 37 (6), 989-990.

Liu, A. J. (2018). Clinical Study on Treatment of Post - Stroke Cognitive Impairment by Tongnao Decoction. Nanjing, China: Nanjing University of Chinese Medicine.

Liu, J. W. (2013). Case Control Study on the Effect of Combination of Tong Qiao Huo Xue Tang and Nimodipine in Treating Vascular Cognitive Impairment after Cerebral Infarction. Guangzhou, China: Guangzhou University of Chinese Medicine.

Liu, L. M. (2015). Effect of Butylphthalide Combined with Buyang Huanwu Decoction on Cognitive Dysfunction of Acute Cerebral Infarction. J. New Chin. Med. 47 (11), 40-41. doi:10.13457/j.cnki.jncm.2015.11.018

Liu, M. (2020). Clinical Efficacy of Shen Rong Tongmai Capsule Combined with Donepezil Hydrochloride Tablets in the Treatment of post-stroke Cognitive Impairment (Deficiency of Kidney Essence). Shanxi, China: Shanxi University of Chinese Medicine.

Liu, S. F., and Liu, H. R. (2005). Treatment of 60 Cases of Dementia after Ischemic Stroke by Invigorating Qi, Activating Blood, Clearing Collaterals, Invigorating Qiao, Awakening Brain and Filling Marrow. J. Sichuan Traditional Chin. Med. 23 (10), 53-54. doi:10.3969/j.issn.1000-3649.2005.10.032

Liu, W., and Yuan, B. (2021). Effect of Self-Designed Huoxue Huayu Xingnao Decoction on MMSE Score and Hemorheology of Patients with post-stroke Cognitive Impairment. Forum Traditional Chin. Med. 36 (2), 28-30. doi:10. 13913/j.cnki.41-1110/r.2021.02.012

Liu, Y. (2014). Effective Observation on Treating Cognitive Functional Disorder Caused by Acute Cerebral Infarction with the Tianzhi Granule Plus Oxiracetam. Clin. J. Chin. Med. 6 (26), 90-91. doi:10.3969/j.issn.1674-7860. 2014.26.053

Liu, Y. J., Jin, F. F., and Chai, J. D. (2020). Effect of Tongqiao Huoxue Decoction Combined with Conventional Treatment on Patients with Cognitive Dysfunction after Acute Ischemic Stroke. Chin. Traditional Patent Med. 42 (7), 1959-1962. doi:10.3969/j.issn.1001-1528.2020.07.057

Liu, Y. Y. (2019). Effect of Yishen Huayu Tongluo Decoction on Vascular Dementia after Cerebral Infarction. Med. J. Chin. People's Health 31 (20), 117-119. doi:10.3969/j.issn.1672-0369.2019.20.050

Luo, D., Wan, X., Liu, J., and Tong, T. (2018). Optimally Estimating the Sample Mean from the Sample Size, Median, Mid-range, And/or Mid-quartile Range. Stat. Methods Med. Res. 27 (6), 1785-1805. doi:10.1177/0962280216669183

Luo, W., and Liu, L. (2016). Clinical Observation of Mild Cognitive Impairment after Ischemic Stroke Treatment Efficacy Flavored Ditan Decoction. Shaanxi J. Traditional Chin. Med. 37 (2), 149-150+154. doi:10.3969/j.issn.1000-7369. 2016.02.007

Ma, X. H., and Zhou, L. H. (2020). A Randomized Parallel Control Trial of Tongnao Yisui Decoction in the Treatment of Mild Cognitive Impairment after Acute Stroke. Xinjiang J. Traditional Chin. Med. 38 (1), 7-9. : CNKI:SUN: XJZY.0.2020-01-003.

Ma, Y., and Lin, J. (2016). Clinical Analysis of Donepezil Combined with Dengzhan Shengmai Capsule in the Treatment of Cognitive Dysfunction in post-stroke Patients. World Clin. Drugs 37 (10), 698-702. doi:10.13683/j.wph.2016.10.009

Mao, J., Huang, S., Liu, S., Feng, X. L., Yu, M., Liu, J., et al. (2015). A Herbal Medicine for Alzheimer's Disease and its Active Constituents Promote Neural Progenitor Proliferation. Aging Cell 14 (5), 784-796. doi:10.1111/acel.12356

Mcgrath, S., Zhao, X., Steele, R., Thombs, B. D., and Benedetti, A. (2020). Estimating the Sample Mean and Standard Deviation from Commonly Reported Quantiles in Meta-Analysis. Stat. Methods Med. Res. 962280219889080, 962280219889080. doi:10.1177/ 0962280219889080

Medeiros, G. C., Roy, D., Kontos, N., and Beach, S. R. (2020). Post-stroke Depression: A 2020 Updated Review. Gen. Hosp. Psychiatry 66, 70-80. doi:10.1016/j.genhosppsych.2020.06.011
Mei, M. F. (2011). A Systematic Analysis of the Theory and Practice of Syndrome Differentiation. Chin. J. Integr. Med. 17 (11), 803-810. doi:10.1007/s11655-0110890-0

Mellon, L., Brewer, L., Hall, P., Horgan, F., Williams, D., and Hickey, A. (2015). Cognitive Impairment Six Months after Ischaemic Stroke: a Profile from the ASPIRE-S Study. BMC Neurol. 15, 31. doi:10.1186/s12883-015-0288-2

Meng, X. J., Cui, W., and Li, A. H. (2020). Clinical Study on Peiyuan Tongnao Capsules Combined with Oxiracetam in Treatment of Vascular Dementia after Stroke. Drugs \& Clinic 35 (6), 1126-1130. doi:10.7501/j.issn.1674-5515.2020.06.015

Mijajlović, M. D., Pavlović, A., Brainin, M., Heiss, W. D., Quinn, T. J., Ihle-Hansen, H. B., et al. (2017). Post-stroke Dementia - a Comprehensive Review. BMC Med. 15 (1), 11. doi:10.1186/s12916-017-0779-7

Mo, H. H. (2010). A Clinical Research for Early Intervention on Mild Cognitive Impairment after Cerebral Infarction by Ziyinjiannao Tablet. Guangzhou, China: Guangzhou University of Chinese Medicine.

Mok, V. C., Lam, B. Y., Wong, A., Ko, H., Markus, H. S., and Wong, L. K. (2017). Early-onset and Delayed-Onset Poststroke Dementia - Revisiting the Mechanisms. Nat. Rev. Neurol. 13 (3), 148-159. doi:10.1038/nrneurol.2017.16

Nijsse, B., Visser-Meily, J. M., van Mierlo, M. L., Post, M. W., de Kort, P. L., and van Heugten, C. M. (2017). Temporal Evolution of Poststroke Cognitive Impairment Using the Montreal Cognitive Assessment. Stroke 48 (1), 98-104. doi:10.1161/STROKEAHA.116.014168

Pendlebury, S. T., and Rothwell, P. M. (2019). Incidence and Prevalence of Dementia Associated with Transient Ischaemic Attack and Stroke: Analysis of the Population-Based Oxford Vascular Study. Lancet Neurol. 18 (3), 248-258. doi:10.1016/S1474-4422(18)30442-3

Price, C. C., Jefferson, A. L., Merino, J. G., Heilman, K. M., and Libon, D. J. (2005). Subcortical Vascular Dementia: Integrating Neuropsychological and Neuroradiologic Data. Neurology 65 (3), 376-382. doi:10.1212/01.wnl. 0000168877.06011.15

Quinn, T. J., Richard, E., Teuschl, Y., Gattringer, T., Hafdi, M., O'Brien, J. T., et al. (2021). European Stroke Organisation and European Academy of Neurology Joint Guidelines on post-stroke Cognitive Impairment. Eur. J. Neurol. 28 (12), 3883-3920. doi:10.1111/ene.15068

Ren, S. H., Zhang, R. J., Liu, J. B., Shi, Y. F., Guo, Y., Cheng, H., et al. (2018). Clinical Observation of Yishen Huayu Decoction Combined with Donepezil Hydrochloride Tablets in the Treatment of Cognitive Dysfunction after Stroke with Kidney Deficiency and Blood Stasis and its Related Mechanism. Hebei J. TCM 40 (3), 390-393. doi:10.3969/j.issn.1002-2619.2018.03.016

Ren, Y., Houghton, P., and Hider, R. C. (2006). Relevant Activities of Extracts and Constituents of Animals Used in Traditional Chinese Medicine for central Nervous System Effects Associated with Alzheimer's Disease. J. Pharm. Pharmacol. 58 (7), 989-996. doi:10.1211/jpp.58.7.0015

Robinson, R. G., and Jorge, R. E. (2016). Post-stroke Depression: A Review. Am. J. Psychiatry 173 (3), 221-231. doi:10.1176/appi.ajp.2015.15030363

Schulz, K. F., Altman, D. G., and Moher, D. (2010). CONSORT 2010 Statement: Updated Guidelines for Reporting Parallel Group Randomised Trials. BMJ 340 (3), c332. doi:10.1136/bmj.c332

Shen, W., Zeng, Z. X., Jin, X. L., Lu, Y., Wang, Y., Li, S. X., et al. (2020). Systematic Review on Clinical Efficacy and Safety of TCM for post Stroke Cognitive Impairment. Chin. J. Exp. Traditional Med. Formulae 26 (11), 185-193. doi:10. 13422/j.cnki.syfjx.20201151

Shi, D., Chen, X., and Li, Z. (2018). Diagnostic Test Accuracy of the Montreal Cognitive Assessment in the Detection of post-stroke Cognitive Impairment under Different Stages and Cutoffs: a Systematic Review and Meta-Analysis. Neurol. Sci. 39 (4), 705-716. doi:10.1007/s10072-018-3254-0

Shi, J., Wei, M., Ni, J., Sun, F., Sun, L., Wang, J., et al. (2020). Tianzhi Granule Improves Cognition and BPSD of Vascular Dementia: a Randomized Controlled Trial. J. Transl. Med. 18 (1), 76. doi:10.1186/s12967-020-02232-z

Song, S. C. (2018). Clinical Curative Effective Research of Shuxuetongmai Granule on Post-Stroke Congnitive Impairment Patient. Guangxi, China: Guangxi University of Chinese Medicine.

Sterne, J. A. C., Savović, J., Page, M. J., Elbers, R. G., Blencowe, N. S., Boutron, I., et al. (2019). RoB 2: a Revised Tool for Assessing Risk of Bias in Randomised Trials. BMJ 366, 14898. doi:10.1136/bmj.14898

Sun, J. H., Tan, L., and Yu, J. T. (2014). Post-stroke Cognitive Impairment: Epidemiology, Mechanisms and Management. Ann. Transl Med. 2 (8), 80. doi:10.3978/j.issn.2305-5839.2014.08.05 
Swartz, R. H., Bayley, M., Lanctôt, K. L., Murray, B. J., Cayley, M. L., Lien, K., et al. (2016). Post-stroke Depression, Obstructive Sleep Apnea, and Cognitive Impairment: Rationale for, and Barriers to, Routine Screening. Int. J. Stroke. 11 (5), 509-518. doi:10.1177/1747493016641968

Tan, L. L., Chen, X. H., Zeng, P. P., He, Q. C., Zhang, Q. P., Hu, Y. Y., et al. (2016). Effect of Shuxue Tongmai Granule on Cognitive Dysfunction after Stroke. Inner Mongolia J. Traditional Chin. Med. 35 (11), 39-40. doi:10.3969/j.issn.10060979.2016.11.038

Teng, Z., Dong, Y., Zhang, D., An, J., and Lv, P. (2017). Cerebral Small Vessel Disease and post-stroke Cognitive Impairment. Int. J. Neurosci. 127 (9), 824-830. doi:10.1080/00207454.2016.1261291

Wan, X., Wang, W., Liu, J., and Tong, T. (2014). Estimating the Sample Mean and Standard Deviation from the Sample Size, Median, Range And/or Interquartile Range. BMC Med. Res. Methodol. 14, 135. doi:10.1186/1471-2288-14-135

Wang, J. (2020a). Expert Consensus on the Prevention and Treatment of PostStroke Cognitive Impairment in China. Chin. J. Stroke 15 (2), 158-166. doi:10. 3969/j.issn.1673-5765.2020.02.010

Wang, K., Dong, Q., Yu, J. T., and Hu, P. P. (2021a). Expert Consensus on the Management of Post-Stroke Cognitive Impairment 2021. Chin. J. Stroke 16 (4), 376-389. doi:10.3969/j.issn.1673-5765.2021.04.011

Wang, L. J., Chen, H. Q., Wang, J. Y., Wang, Y., and Zhu, H. M. (2021b). Clinical Effects of Modified Chaimu Xingnao Decoction Combined with Oxiracetam on Patients with Cognitive Dysfunction of Senile Stroke. Chin. Traditional Patent Med. 43 (1), 89-91. doi:10.3969/j.issn.1001-1528.2021.01.017

Wang, L., Zhang, J. J., Ding, X. Y., Wang, Y. L., Lang, F., Li, Q., et al. (2018). Effect of Buqi Huoxue Yizhi Therapy on Mild Cognitive Impairment (None Dementia) after Ischemic Stroke. Mod. Chin. Clin. Med. 25 (2), 18-21. doi:10.3969/j.issn. 2095-6606.2018.02.00610.1111/ene.13497

Wang, Y. W. (2020b). Clinical Study on the Treatment of Cognitive Impairment in the Acute Stage of Ischemic Stroke with Zhong Feng Xing Nao Liquid. Chengdu, China: Chengdu University of Traditional Chinese Medicine.

Wu, Y., Hu, S., Ma, Y., Zhao, B., Yang, W., Lu, Y., et al. (2020). Novel Pheretima Guillelmi-Derived Antithrombotic Protein DPf3: Identification, Characterization, In Vitro Evaluation and Antithrombotic Mechanisms Investigation. Int. J. Biol. Macromol. 154, 545-556. doi:10.1016/j.ijbiomac. 2020.03.097

Xin, J., Zhang, J., Yang, Y., Deng, M., and Xie, X. (2013). Radix Angelica Sinensis that Contains the Component Z-Ligustilide Promotes Adult Neurogenesis to Mediate Recovery from Cognitive Impairment. Curr. Neurovasc. Res. 10 (4), 304-315. doi:10.2174/15672026113109990023

Xing, S. H., Zhu, C. X., Zhang, R., and An, L. (20142014). Huperzine a in the Treatment of Alzheimer's Disease and Vascular Dementia: a Meta-Analysis. Evid. Based Complement. Alternat Med. 2014, 363985. doi:10.1155/2014/ 363985

Xu, P., Liang, L., and Bai, X. (2019). Study on the Clinical Effect of Modified Qufengtongqiao Prescription on the Post-stroke Cognitive Impairment. World Latest Med. Inf. 19 (96), 16-17+19. doi:10.19613/j.cnki.1671-3141.2019.96.008

Xu, S. Z., Yang, H. Y., Tian, J. B., Yang, Z. H., Li, Y. Z., and Zhu, J. W. (2014). Treatment of Mild Cognitive Function Impairment after Stroke with Qianglizengzhi Drink. Hebei J. TCM 36 (10), 1455-1457. doi:10.3969/j.issn. 1002-2619.2014.10.007
Xu, Y. J., Mei, Y., Qu, Z. L., Zhang, S. J., Zhao, W., Fang, J. S., et al. (2018). Ligustilide Ameliorates Memory Deficiency in APP/PS1 Transgenic Mice via Restoring Mitochondrial Dysfunction. Biomed. Res. Int. 2018, 4606752. doi:10. $1155 / 2018 / 4606752$

Xu, Z. Q., Liang, X. M., Wu, J., Zhang, Y. F., Zhu, C. X., and Jiang, X. J. (2012). Treatment with Huperzine A Improves Cognition in Vascular Dementia Patients. Cell Biochem. Biophys. 62 (1), 55-58. doi:10.1007/s12013-0119258-5

Yang, S. Q., Fan, D. H., and Lin, H. G. (2021). Clinical Study of Xiongqi Xingnao Recipe Combined with Butylphthalide in Vascular Cognitive Impairment Caused by Ischemic Stroke. Chin. Manipulation Rehabil. Med. 12 (13), 34-38. doi:10.19787/j.issn.1008-1879.2021.13.011

Yao, S. D. (2020). Analysis of Medication Rule of post-stroke Cognitive Impairment in Modern Literature Based on TCM Inheritance Assistance Platform. Liaoning, China: Liaoning University Of Traditional Chinese Medicine.

You, Y. Y. (2019). Clinical Research of Yiqishuxuetongmai Prescription on Patients with Cognitive Impairment after Ischemic Stroke of Qi Deficiency and Sputum Stasis. Guangxi, China: Guangxi University of Chinese Medicine.

Zhang, X., and Bi, X. (2020). Post-stroke Cognitive Impairment: A Review Focusing on Molecular Biomarkers. J. Mol. Neurosci. 70 (8), 1244-1254. doi:10.1007/s12031-020-01533-8

Zhang, X., Yuan, M., Yang, S., Chen, X., Wu, J., Wen, M., et al. (2021). Enriched Environment Improves post-stroke Cognitive Impairment and Inhibits Neuroinflammation and Oxidative Stress by Activating Nrf2-ARE Pathway. Int. J. Neurosci. 131 (7), 641-649. doi:10.1080/00207454.2020.1797722

Zhong, H. H., Qu, J. F., Xiao, W. M., Chen, Y. K., Liu, Y. L., Wu, Z. Q., et al. (2021). Severity of Lesions Involving the Cortical Cholinergic Pathways May Be Associated with Cognitive Impairment in Subacute Ischemic Stroke. Front. Neurol. 12, 606897. doi:10.3389/fneur.2021.606897

Zhuo, J. M., Wan, Z. Q., and Lu, L. C. (2016). Effect of Butylphthalide Combined with Yangxue Qingnao Granule on Cognitive Dysfunction after Stroke. Chin. J. Rural Med. Pharm. 23 (9), 32-33. doi:10.3969/j. issn.1006-5180.2016.09.019

Conflict of Interest: The authors declare that the research was conducted in the absence of any commercial or financial relationships that could be construed as a potential conflict of interest.

Publisher's Note: All claims expressed in this article are solely those of the authors and do not necessarily represent those of their affiliated organizations, or those of the publisher, the editors and the reviewers. Any product that may be evaluated in this article, or claim that may be made by its manufacturer, is not guaranteed or endorsed by the publisher.

Copyright (c) 2022 Shen, Fan, Wang and Zhang. This is an open-access article distributed under the terms of the Creative Commons Attribution License (CC BY). The use, distribution or reproduction in other forums is permitted, provided the original author(s) and the copyright owner(s) are credited and that the original publication in this journal is cited, in accordance with accepted academic practice. No use, distribution or reproduction is permitted which does not comply with these terms. 